\title{
Free and Forced Components of Shoaling Long Waves in the Absence of Short-Wave Breaking $\mathscr{O}$
}

\author{
StePhanie Contardo, ${ }^{\mathrm{a}, \mathrm{b}}$ Ryan J. Lowe, ${ }^{\mathrm{b}, \mathrm{c}}$ JefF E. HANSen, ${ }^{\mathrm{b}}$ Dirk P. Rijnsdorp,${ }^{\mathrm{c}, \mathrm{d}}$ FrançOis Dufois, ${ }^{\mathrm{e}}$ And \\ GRAHAM SYMONDS ${ }^{\mathrm{b}}$ \\ ${ }^{a}$ CSIRO Oceans and Atmosphere, Crawley, Western Australia, Australia \\ ${ }^{\mathrm{b}}$ School of Earth Sciences, University of Western Australia, Crawley, Western Australia, Australia \\ ${ }^{\mathrm{c}}$ Oceans Graduate School, University of Western Australia, Crawley, Western Australia, Australia \\ ${ }^{\mathrm{d}}$ Environmental Fluid Mechanics Section, Faculty of Civil Engineering and Geosciences, Delft University of Technology, Delft, \\ Netherlands \\ e IFREMER, DYNECO/DHYSED, Plouzané, France
}

(Manuscript received 2 September 2020, in final form 8 February 2021)

\begin{abstract}
Long waves are generated and transform when short-wave groups propagate into shallow water, but the generation and transformation processes are not fully understood. In this study we develop an analytical solution to the linearized shallow-water equations at the wave-group scale, which decomposes the long waves into a forced solution (a bound long wave) and free solutions (free long waves). The solution relies on the hypothesis that free long waves are continuously generated as short-wave groups propagate over a varying depth. We show that the superposition of free long waves and a bound long wave results in a shift of the phase between the short-wave group and the total long wave, as the depth decreases prior to short-wave breaking. While it is known that short-wave breaking leads to free-long-wave generation, through breakpoint forcing and bound-wave release mechanisms, we highlight the importance of an additional freelong-wave generation mechanism due to depth variations, in the absence of breaking. This mechanism is important because as free long waves of different origins combine, the total free-long-wave amplitude is dependent on their phase relationship. Our free and forced solutions are verified against a linear numerical model, and we show how our solution is consistent with prior theory that does not explicitly decouple free and forced motions. We also validate the results with data from a nonlinear phase-resolving numerical wave model and experimental measurements, demonstrating that our analytical model can explain trends observed in more complete representations of the hydrodynamics.
\end{abstract}

KEYWORDS: Ocean dynamics; Shallow-water equations; Wind waves

\section{Introduction}

As short-wave groups propagate into intermediate or shallow water, they are accompanied by long waves with the same frequency as the group, which are excited through nonlinear interactions between all possible pairs of short-wave frequencies. These "bound" waves were first described theoretically by LonguetHiggins and Stewart (1962) as a response to gradients in radiation stress at the scale of short-wave groups. Their analytical solution was based on wave propagation in constant depth, in which case the bound waves were locked and $180^{\circ}$ out of phase with the group. In varying depth, the phase relationship between the group and the long wave has been observed to change, and in progressively shallower water, the growth rate of the long waves is smaller than expected according to the equilibrium solution for a constant depth (Elgar et al. 1992; Battjes et al. 2004; van Dongeren et al. 2007).

While Longuet-Higgins and Stewart (1962) did not provide an analytical solution for wave groups propagating over varying depth, several subsequent mathematical and numerical modeling studies (Janssen et al. 2003, hereinafter J03; van Leeuwen 1992;

Supplemental information related to this paper is available at the Journals Online website: https://doi.org/10.1175/JPO-D-200214.s1.

Corresponding author: Stephanie Contardo, stephanie.contardo@ csiro.au
Mei and Benmoussa 1984; List 1992; Okihiro et al. 1992; van Dongeren et al. 2003; Schäffer 1993; Roelvink et al. 1993) as well as laboratory experiments (Battjes et al. 2004; van Noorloos 2003) were conducted to investigate the hydrodynamic processes. These studies have consistently shown that long waves increasingly lag the wave group as they advance into shallower water, approaching a phase lag of $90^{\circ}$ as the depth tends to zero. Li et al. (2020) also noted that the amplitude of long waves was still increasing on the plateau of a shoal when depth variations ceased, highlighting the importance of accounting for "wave history" to investigate long-wave transformation. While mathematical and numerical models have been able to provide reasonable estimates of the amplitude and phase of long waves in the nearshore (J03; van Dongeren et al. 2003; van Leeuwen 1992; Zou 2004), these modeling studies have not yet provided a complete physical explanation for the long-wave transformation across the surf zone (Bertin et al. 2018, 2020). For instance, the perturbation approach chosen by $\mathrm{J} 03$ provides a solution very close to the exact solution calculated through variation of parameters (van Leeuwen 1992; Schäffer 1993). While these mathematical models are able to reproduce the phase shift and estimate the wave growth, they do not provide a complete physical explanation for why there is a phase shift, and why the observed growth rate is larger than the growth rate of shoaling free waves (i.e., from Green's law) but less than the growth predicted by the equilibrium bound wave solution (Longuet-Higgins and Stewart 1962).

Hypotheses have been proposed in the literature to explain the growth of long waves in the nearshore. J03 suggested that 
the positive part of the correlation between wave groups and long waves was due to free waves, referring to Molin (1982) and Mei and Benmoussa (1984), who described the generation of free long waves by depth variations. Nagase and Mizuguchi (1997) also made similar arguments to explain free-wave generation during the shoaling process, based on laboratory observations. More recently, Nielsen and Baldock (2010) proposed a qualitative explanation for the phase shift by considering the forcing of a transient long wave generated by a single short-wave group. In a more recent paper (Nielsen 2017), the Nielsen and Baldock (2010) flat bottom model was used to offer an intuitive explanation for the case of a shoaling wave group. Nielsen (2017) explained how the generation of successive incremental free long waves, in addition to the bound long wave, is required to allow for mass conservation as a transient short-wave group propagates over a sloping bottom. These results are supported by the numerical model results of Moura and Baldock (2019) that highlight the presence of free waves in the bound-long-wave shoaling process. The earlier results presented by Lara et al. (2010) using a numerical model and laboratory experiment are also qualitatively consistent with the long-wave growth process of Nielsen (2017). However, their numerical study, based on the Reynolds averaged Navier-Stokes equations which includes wave breaking and dissipation, did not allow them to isolate the mechanisms associated with depth variation. Thus, despite increased attention, the transformation of long waves as nonbreaking wave groups propagate over varying bathymetry is still not yet fully explained.

In contrast, there are two established mechanisms for the case where wave groups break in shallow water, which have been used to describe the generation of free long waves: bound-wave release (Inch et al. 2017; Masselink 1995) and breakpoint forcing (Symonds et al. 1982; Contardo et al. 2018; Contardo and Symonds 2013, 2016; Moura and Baldock 2017; Pomeroy et al. 2012). While these additional mechanisms relating specifically to breaking are very important to long-wave generation in the nearshore, in this study we focus on an alternative generation mechanism of free long waves by nonbreaking wave groups interacting with depth variations outside of the breaking region.

In this study, we propose a solution to the linearized shallowwater equations at the short-wave group scale for the case of a sloping bottom, which decomposes the water level into free and forced components. Our total solution (addition of the free and forced components) agrees with the results of a linearized numerical model resolving the governing mass-momentum equations and with J03's solution, obtained with a perturbation approach, but provides new insight into the (de)coupling of the free and forced contributions. This demonstrates the consistency of the approach and supports the physical interpretation of the underlying mechanisms. Finally, we validate the linearized results (both analytical and numerical model) with results from a nonhydrostatic phase-resolving wave-flow model Simulating Waves till Shore (SWASH; Zijlema et al. 2011; Rijnsdorp et al. 2014) as well as with laboratory wave flume measurements. The validation supports that the linearized model can explain the trends observed in more complete representations of the hydrodynamics.

\section{Background}

The depth-integrated mass and momentum conservation equations, averaged over short-wave periods, can be expressed as (e.g., Schäffer 1993)

$$
\begin{aligned}
& \frac{\partial \zeta}{\partial t}+\frac{\partial[(h+\zeta) U]}{\partial x}=0 \\
& \frac{\partial U}{\partial t}+U \frac{\partial U}{\partial x}+g \frac{\partial \zeta}{\partial x}=-\frac{1}{\rho(h+\zeta)} \frac{\partial S_{x x}}{\partial x}
\end{aligned}
$$

where $x$ is the cross-shore coordinate (positive offshore) with the origin at the shoreline, $t$ is the time, $h$ is the still water depth, $U$ is the depth-integrated current velocity, $\zeta$ the sea surface elevation relative to still water level, $\rho$ the seawater density, and $g$ the gravitational acceleration. The term $S_{x x}$ is the radiation stress (excess momentum flux due to the short waves) averaged over the short-wave time scale that retains the wave-group time scale:

$$
S_{x x}=\frac{1}{2} \rho g A_{g}^{2}\left(\frac{2 c_{g}}{c}-\frac{1}{2}\right),
$$

where $A_{g}(x, t)$ is the wave group envelope, $c_{g}(x)$ is the group velocity, and $c(x)$ the phase velocity of the short waves. Equations (1) and (2) represent the nonlinear shallow-water equations with a forcing term incorporated in the momentum equation. Assuming $\zeta \ll h$, depths are slowly varying (mild slopes) and neglecting nonlinear terms, we obtain the linearized shallow-water equations (Longuet-Higgins and Stewart 1962; Schäffer 1993):

$$
\begin{aligned}
& \frac{\partial \zeta}{\partial t}+h \frac{\partial U}{\partial x}=0, \\
& \frac{\partial U}{\partial t}+g \frac{\partial \zeta}{\partial x}=-\frac{1}{\rho h} \frac{\partial S_{x x}}{\partial x} .
\end{aligned}
$$

By cross-differentiating Eqs. (4) and (5), $U$ is eliminated and we obtain (Longuet-Higgins and Stewart 1962; Schäffer 1993; Mei and Benmoussa 1984)

$$
\frac{\partial}{\partial x}\left(g h \frac{\partial \zeta}{\partial x}\right)+\frac{\partial^{2} \zeta}{\partial t^{2}}=-\frac{1}{\rho} \frac{\partial^{2} S_{x x}}{\partial x^{2}} .
$$

In the particular case of a flat bottom, this equation has a simple analytical solution (Longuet-Higgins and Stewart 1962), which gives the classic solution for the bound long wave (BLW) surface elevation and current velocity:

$$
\begin{gathered}
\zeta_{\mathrm{BLW}}=-\frac{1}{\rho}\left(\frac{S_{x x}}{g h-c_{g}^{2}}\right)+\text { constant } \\
U_{\mathrm{BLW}}=-\frac{1}{\rho h}\left(\frac{c_{g} S_{x x}}{g h-c_{g}^{2}}\right)+\text { constant } .
\end{gathered}
$$

This solution is valid under the assumption that long waves are in shallow water and has been validated in many studies (e.g., Battjes et al. 2004; van Dongeren et al. 2007; Herbers et al. 1994; Lara et al. 2010; Torres-Freyermuth et al. 2010). In this paper, we will refer to the BLWs as the flat bottom solution of LonguetHiggins and Stewart (1962). In contrast, the solution over varying 
bathymetry, sometimes referred to as "shoaling bound wave" (e.g., Battjes et al. 2004), will be referred to as the total long wave (TLW), as we show below that the BLW (the flat bottom solution) is the only component of the TLW that is strictly bound to the group and propagates at the group velocity and out of phase with the wave group envelope, with the rest of the TLW signal being FLWs generated as the wave groups propagates over varying bathymetry.

\section{Analytical approach}

Here we formulate a solution to the linearized shallow-water equations [Eqs. (4) and (5)] over a variable depth profile. Our solution is based on the hypothesis that the total long wave is a superposition of a BLW and FLWs as suggested in the literature (Mei and Benmoussa 1984; Moura and Baldock 2019; Nielsen 2017; Zhang et al. 2020). Our objective is to calculate the long-wave amplitude and phase relationship with the short-wave group, as the wave group propagates over a sloping bottom. We start with the simple case of a single depth variation (a single step) and then extend this to a slope which we treat as a series of infinitesimally small steps.

Here we provide expressions for a single-frequency long wave, representing the difference interactions between two short-wave frequencies within a spectral group. Since the process would be repeated for every pair of short-wave frequency interactions, the amplitude of a spectrum of long waves associated with an irregular short-wave spectrum can be estimated by integrating all of the low-frequency contributions (Herbers et al. 1994; Ruessink 1998; Reniers et al. 2002; van Dongeren et al. 2003).

\section{a. Single step case}

We first calculate the amplitude of the incoming and outgoing FLWs (denoted iFLW and oFLW, respectively) generated over a single depth discontinuity (a single step). The step is treated as a boundary between two zones: seaward and shoreward. We calculate the elevation amplitude of the FLWs, as a function of the elevation amplitude of the BLWs, by matching the elevation and momentum solutions along the boundary of the seaward and shoreward zones (e.g., Symonds et al. 1982; Schäffer 1993). The continuity of the long-wave elevation $\left(\zeta_{\mathrm{LW}}\right)$ and momentum $\left(M_{\mathrm{LW}}\right)$ at the bathymetric interface implies that at the step $\left(x=x_{0}\right)$ :

$$
\zeta_{\mathrm{BLW}, \mathrm{sea}}\left(x_{0}, t\right)+\zeta_{\mathrm{oFLW}}\left(x_{0}, t\right)=\zeta_{\mathrm{BLW}, \text { shore }}\left(x_{0}, t\right)+\zeta_{\mathrm{iFLW}}\left(x_{0}, t\right),
$$

and

$$
\begin{aligned}
M_{\mathrm{BLW}, \text { sea }}\left(x_{0}, t\right)+M_{\mathrm{oFLW}}\left(x_{0}, t\right)= & M_{\mathrm{BLW}, \text { shore }}\left(x_{0}, t\right) \\
& +M_{\mathrm{iFLW}}\left(x_{0}, t\right) .
\end{aligned}
$$

The BLWs are in antiphase with the group [Eq. (7)] and we hypothesize that the iFLW and the oFLW are respectively in antiphase and in phase with the BLW (this will be verified in section 4a). We make this hypothesis because the generation of the FLWs coincides with the propagation of the wave group (and the BLW) over the step, similar to the "abrupt onset" described by Nielsen and Baldock (2010) where free waves are generated to satisfy the flat surface condition when the radiation stress forcing is instantaneously switched on: $\zeta_{\text {iFLW }}+\zeta_{\text {ofLW }}+$ $\zeta_{\mathrm{BLW}}=0$. Based on these assumptions, the total surface elevation on the seaward and shoreward sides can be written, respectively, as

$$
\begin{aligned}
\zeta_{\text {sea }}= & A_{\mathrm{BLW}, \text { sea }} \cos \left[k_{b, \text { sea }}\left(x-x_{0}\right)-\omega t\right] \\
& +A_{\mathrm{oFLW}} \cos \left[k_{\mathrm{f}, \mathrm{sea}}\left(x-x_{0}\right)+\omega t\right]
\end{aligned}
$$

and

$$
\begin{aligned}
\zeta_{\text {shore }}= & A_{\mathrm{BLW}, \text { shore }} \cos \left[k_{b, \text { shore }}\left(x-x_{0}\right)-\omega t\right] \\
& -A_{\mathrm{iFLW}} \cos \left[k_{f, \text { shore }}\left(x-x_{0}\right)-\omega t\right] .
\end{aligned}
$$

The momentum is described by $M=\rho h U$ (as we have made the assumption $\zeta \ll h$; Schäffer 1993), so the total momentum, on the seaward and shoreward sides can be written, respectively,

$$
\begin{aligned}
M_{\text {sea }}= & \frac{\rho g h_{\text {sea }}}{\omega}\left\{k_{b, \text { sea }} A_{\mathrm{BLW}, \mathrm{sea}} \cos \left[k_{b, \text { sea }}\left(x-x_{0}\right)-\omega t\right]\right. \\
& \left.-k_{f, \text { sea }} A_{\text {ofLW }} \cos \left[k_{f, \text { sea }}\left(x-x_{0}\right)+\omega t\right]\right\}
\end{aligned}
$$

and

$$
\begin{aligned}
M_{\text {shore }}= & \frac{\rho g h_{\text {shore }}}{\omega}\left\{k_{b, \text { shore }} A_{\mathrm{BLW}, \text { shore }} \cos \left[k_{b, \text { shore }}\left(x-x_{0}\right)-\omega t\right]\right. \\
& \left.-k_{f \text {.shore }} A_{\text {ifLW }} \cos \left[k_{f, \text { sea }}\left(x-x_{0}\right)-\omega t\right]\right\},
\end{aligned}
$$

where $\omega$ is the long-wave angular frequency (which is equal to the group angular frequency); $A_{\mathrm{iFLW}}$ and $A_{\mathrm{oFLW}}$ are the amplitudes of the incoming (shoreward propagating) and outgoing (seaward propagating) FLWs, respectively; $A_{\mathrm{BLW}}$,sea and $A_{\mathrm{BLW}}$,shore are the amplitudes of the BLW; $k_{b \text {,sea }}$ and $k_{b \text {,shore }}$ are the wavenumbers of the BLW; $k_{f \text {,sea }}$ and $k_{f \text {,shore }}$ are the wavenumbers of the FLW; and $h_{\text {sea }}$ and $h_{\text {shore }}$ are the depths, seaward and shoreward of the step, respectively. We then match the solutions at $x=x_{0}$ to obtain the constants $A_{\mathrm{iFLW}}, A_{\mathrm{oFLW}}, A_{\mathrm{BLW} \text {,shore }}$, and $A_{\mathrm{BLW}, \text { sea. }}$ Equations (9) and (10) then become

$$
A_{\mathrm{BLW}, \mathrm{sea}}+A_{\mathrm{oFLW}}=A_{\mathrm{BLW}, \text { shore }}-A_{\mathrm{iFLW}},
$$

and

$$
\begin{aligned}
k_{b, \text { sea }} h_{\text {sea }} A_{\text {BLW,sea }}-k_{f, \text { sea }} h_{\text {sea }} A_{\text {oFLW }}= & k_{b, \text { shore }} h_{\text {shore }} A_{\text {BLW,shore }} \\
& -k_{f, \text { shore }} h_{\text {shore }} A_{\text {iFLW }} .
\end{aligned}
$$

Using this system of equations, we find solutions for $A_{\mathrm{iFLW}}$ and $A_{\mathrm{oFLW}}$ :

$$
A_{\mathrm{iFLW}}=\frac{-A_{\mathrm{BLW}, \text { sea }}\left(k_{f, \text { sea }}+k_{b \text {,sea }}\right) h_{\text {sea }}+A_{\mathrm{BLW} \text {,shore }}\left(k_{f, \text { sea }} h_{\text {sea }}+k_{b, \text { shore }} h_{\text {shore }}\right)}{k_{f, \text { shore }} h_{\text {shore }}+k_{f, \text { sea }} h_{\text {sea }}},
$$




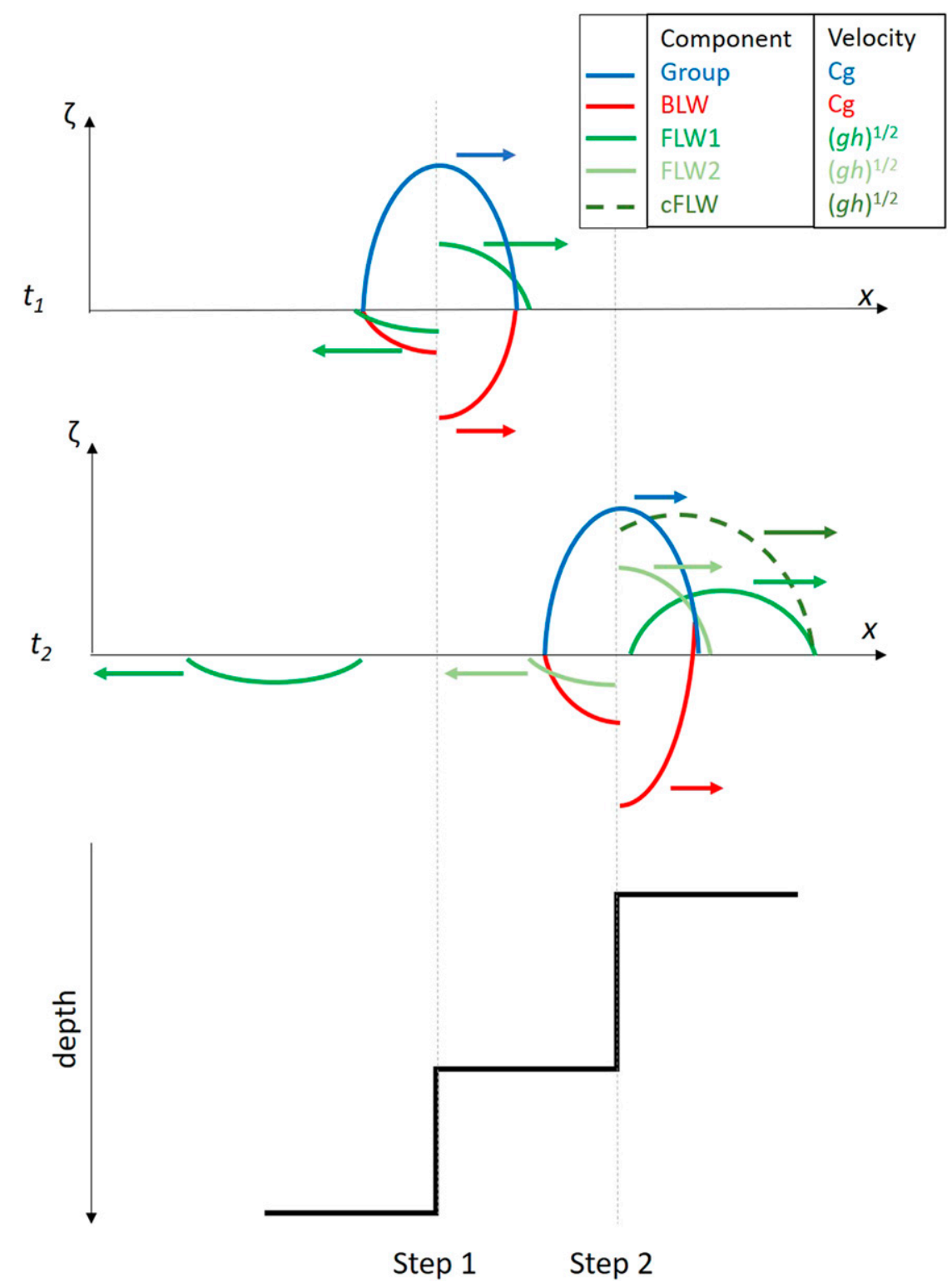

FIG. 1. Schematic description of the generation of FLWs at step 1 (at time $t_{1}$ ) and step 2 (at time $t_{2}$ ). The dashed green line represents the cFLW (iFLW1 and iFLW2 combined). In this figure, we do not represent short-wave shoaling.

$$
A_{\text {oFLW }}=\frac{A_{\text {BLW,sea }}\left(-k_{f, \text { shore }} h_{\text {shore }}+k_{b, \text { sea }} h_{\text {sea }}\right)+A_{\text {BLW,shore }}\left(k_{f, \text { shore }}-k_{b, \text { shore }}\right) h_{\text {shore }}}{k_{f, \text { shore }} h_{\text {shore }}+k_{f, \text { sea }} h_{\text {sea }}} .
$$

\section{b. Slope case}

For the case of a seabed with constant slope (i.e., a plane beach), which can be represented as a succession of infinitesimally small steps, the process we described for the single step case repeats for each depth increment. Therefore, the process where a single iFLW and smaller oFLW are generated in the step case, repeatedly occurs along the slope. The progression of the group over the first depth variation (a step of length $d x$ ) is schematically represented in Fig. 1, showing the initial iFLW and oFLW generated at this initial time $t_{1}$. The propagation over the second depth change is also represented at $t_{2}$. The process is the same, except that at $t_{2}$, an iFLW and an oFLW already exist (generated earlier at $t_{1}$ ). This initial iFLW is now ahead of the group and overlaps with a larger newly generated iFLW. These overlapping iFLWs form a combined FLW (denoted cFLW). The oFLW generated at $t_{1}$ does 
not overlap with the newly generated oFLW, as it propagates away from the group into deeper water. Since the successive oFLWs do not overlap and the oFLWs are smaller than the iFLW, their contribution to the total FLW amplitude is negligible, as will be confirmed by the numerical model in section 4 .

Based on this approach, we can calculate the amplitude of the cFLW as a lagged summation of the amplitude of the iFLWs generated successively at each small single step of the slope. The expression of the amplitude of the cFLW for the slope case is, therefore, based on the superposition of the amplitude and phases of the iFLW generated in the single step case [Eq. (17)]. At each grid cell, the amplitude of the cFLW is the combined amplitude of the shoaling lagged FLW:

$$
A_{\mathrm{cFLW}, n x}=\sum_{n w=1}^{n x} A_{\mathrm{FLW}, n w}\left(\frac{g h_{n w}}{g h_{n x}}\right)^{1 / 4} \cos \varphi_{\mathrm{FLW}, n w, n x}
$$

where $n x$ represents the grid cell number under consideration, $n w$ refers to each individual FLW generated (i.e., $n w=1$ is generated at $n x=1, n w=2$ at $n x=2$, etc), and $A_{\mathrm{FLW}, n w}$ is the amplitude of the wave $n w$ generated at $n x=n w$. Here $\varphi_{\mathrm{FLW}, n w, n x}$ is the phase lag between each individual FLW and the group present at grid cell $n x$ :

$$
\varphi_{\mathrm{FLW}, n w, n x}=\omega d x \sum_{i=n w}^{n x}\left(\frac{1}{{\sqrt{g h_{i}}}}-\frac{1}{c_{g, i}}\right) .
$$

Given that the oFLWs are negligible, we only need one equation [Eq. (9)] to calculate the amplitude of the individual iFLWs. The amplitude of each iFLW is calculated as in the single step case. Each grid cell has its own set of boundary conditions, and the amplitude of the FLW generated is obtained using Eq. (17) as

$$
A_{\mathrm{FLW}, n w}=\left[-A_{\mathrm{BLW}, n w-1}\left(k_{f, n w-1}+k_{b, n w-1}\right) h_{n w-1}+A_{\mathrm{BLW}, n w}\left(k_{f, n w-1} h_{n w-1}+k_{b, n w} h_{n w}\right)\right] \frac{1}{\left(k_{f, n w} h_{n w}+k_{f, n w-1} h_{n w-1}\right)} .
$$

In Eq. (21), the amplitude of the BLW ( $\left.A_{\mathrm{BLW}}\right)$ is calculated from the amplitude of the short-wave group based on Eqs. (3) and (7). Hence, this set of equations [Eqs. (19)(21)] can be forced by a wave group of specified amplitude. Using a constant short-wave amplitude is useful, as we show below, to highlight the effect of depth variations on longwave generation. However, shoaling of the short-wave groups can readily be accounted for in Eq. (3). For realistic cases (measured or numerically simulated) short-wave amplitudes may also be used. This allows for comparison with laboratory data and hydrodynamic model results. This is the approach we take in the paper to validate our analytical model (see section 6).

\section{Verification of the analytical approach with full numerical solutions}

In the previous section, we formulated the analytical solutions to the linearized shallow-water equations [Eqs. (4) and (5)]. Here we verify that these solutions are robust and that the assumptions made are reasonable by comparing the analytical solutions to full numerical solutions. Through this application, we also use the results to examine the responses of the long waves in further detail.

The model is based on numerically solving Eqs. (4) and (5) using a finite difference scheme in the $x$ (cross-shore) direction, analogous to the model described in List (1992). We obtain the total surface elevation of the long waves generated, which we refer to as the TLW. In appendix A, we confirm that the numerical model conserves total wave energy, including the exchange of energy between short-wave groups and long waves.

The model is forced with a single (transient) wave group, resulting in radiation stresses at the wave-group time scale
[Eq. (3)]. Such a transient wave group (as opposed to repeated periodic wave groups) allows for a straightforward visualization of the individual FLW and BLW components.

At $t>0$, the single wave group starts propagating from the offshore boundary such that the initial conditions, at $t=0$, for $\zeta$ and $M$ are zero. The single wave group is chosen to have a bichromatic form, representing one pair of short-wave frequencies. From trigonometry the amplitude of the group envelope is

$$
A_{g}(x, t)=\left\{a_{1}^{2}+a_{2}^{2}+2 a_{1} a_{2} \cos [\varphi(x)-\omega t]\right\}^{1 / 2},
$$

where $a_{1}$ and $a_{2}$ are the amplitudes of the two short waves that form the bichromatic wave group and $\varphi$ is the phase function, used to define the local wavenumber. Here $\varphi$ varies with $x$ due to the depth variations and is related to the group wavenumber $\left(k_{b}\right)$ as in $\mathrm{J} 03$ :

$$
\frac{d \varphi}{d x}=k_{b}(x)
$$

At the offshore boundary, the momentum $M$ and surface elevation $\zeta$ are initially prescribed using the flat-bottom analytical solution [Eqs. (7) and (8)]. There is no incoming FLW at the offshore boundary. This is equivalent to assuming that there is a flat-bed section preceding the slope or that the FLW generated outside the domain are negligible, which is reasonable to assume since the depth variations are small compared to the total depth.

We apply the model in two configurations: 1) a single step case, with a deeper region on the seaward side, and 2) a slope case where the depth decreases linearly with the slope $\beta$. The model runs are stopped before the wave group reaches the shoreline boundary $(h=0)$ so we can focus on the incoming signal. 
TABLE 1. Characteristics of step and slope examples using the linear model runs.

\begin{tabular}{lcccccccc}
\hline \hline Case & $a_{1}(\mathrm{~m})$ & $f(\mathrm{~Hz})$ & $f_{l}(\mathrm{~Hz})$ & $h_{\max }(\mathrm{m})$ & $h_{\min }(\mathrm{m})$ & $\beta$ & $d x(\mathrm{~m})$ & $D t(\mathrm{~s})$ \\
\hline Step & 1.0 & 0.010 & 0.125 & 10 & 5 & 1 & 5 & 0.1 \\
Slope & 1.0 & 0.010 & 0.125 & 50 & 0 & 0.01 & 5 & 0.1 \\
\hline
\end{tabular}

As illustrative examples of each case, for the step case we consider a bathymetry where the step transitions from a depth of 10 to $5 \mathrm{~m}$, and for the slope case, where the slope is $\beta=0.05$. The bichromatic group is composed of two interacting $1 \mathrm{~m}$ short waves of frequencies $f_{1}=0.125 \mathrm{~Hz}$ and $f_{2}=0.115 \mathrm{~Hz}$, such that the group frequency difference $(f=f 1-f 2)$ is $0.01 \mathrm{~Hz}$ (Table 1 ). While we focus on this specific set of parameters in this example, we verify that the results display the same general responses, including good agreement between the analytical model and the numerical model, when considering a range of other parameter values (see appendix B). The time step $d t$ is taken as $0.1 \mathrm{~s}$ and the spatial step in the cross-shore direction $d x$ is variable, taken as $5 \mathrm{~m}$ at the offshore boundary and adjusted so that $d x / L$ remains constant throughout the domain, while satisfying the Courant condition to ensure stability. We note that different gridcell sizes were initially tested ( $d x$ between 1 and $20 \mathrm{~m}$ ) and we found the amplitude and phase lag responses were negligibly affected (not shown).

While the runs are set in dimensional variables, we will present the results using nondimensional parameters. Spatial parameters are scaled by the group wavelength $L$. Thus, the nondimensional depth is $h^{*}=h / L$, the nondimensional cross-shore distance is $x^{*}=\left(x_{0}-x\right) / L$, with $x_{0}$ a reference cross-shore location, and the nondimensional amplitude is $A^{*}=A / L$. The temporal parameters are scaled by the wave group angular frequency, i.e., $t^{*}=\omega t$. The nondimensional radiation stress is defined as $S^{*}=$ $S_{x x} / \rho g L^{2}$.

\section{a. Single step case}

In the single step configuration, we aim to isolate the processes responsible for the generation of an iFLW and an oFLW for a given finite depth change. By applying the numerical model in this configuration, we obtain values of elevation and velocity at the group scale. In Fig. 2, we represent the wave group envelope and the surface elevation of the resulting long waves when a single wave group propagates over an initially flat bottom $\left(h_{\mathrm{sea}}^{*}, x^{*}<0\right)$, which then interacts with a step at $x^{*}=0$, where the depth changes to $h_{\text {shore }}^{*}\left(h_{\text {shore }}^{*}<h_{\text {sea }}^{*}, x^{*}>0\right)$. While the amplitude of the group is constant over the domain (in this simplified model), the nondimensional amplitude varies with $L$ and therefore with $h$. The surface elevation of the TLW (Fig. 2b) is obtained from the numerical model and the surface elevation of the BLW (Fig. 2c) is calculated using Eq. (7). As the group propagates within the deeper region prior to reaching the step $\left(x^{*}<0\right)$, the TLW observed is equivalent to the "flat bottom" solution only [Eqs. (7) and (8)], i.e., the BLW is "locked" to the wave group, such that TLW $=$ BLW (Figs. 2b,c). After the step $\left(x^{*}>0\right)$, the TLW is composed of a negative signal preceded by a positive signal (Fig. 2b). We decompose this signal by removing the BLW signal from the
TLW signal (Fig. 2d). The decomposition shows that the positive signal observed in Fig. $2 b$ corresponds to a FLW propagating at $\sqrt{g h}$ (Fig. 2d). The pattern of the TLW arises in this region due to the superposition of a negative signal, representing the BLW (Fig. 2c), and a positive signal (Fig. 2d) in the shallower region. In the deeper region $\left(x^{*}<0\right)$, a negative signal is also present (Figs. 2b,d), propagating as a free wave away from the step. This is the signal of the oFLW, which is small compared to the iFLW. The numerical simulation thus shows a shoreward- and a seaward-propagating FLWs propagating away from the step as the short-wave group propagates over the step, as was hypothesized earlier in section $3 \mathrm{a}$.

We investigate the role of the radiation stress in the elevation of long waves by comparing time series of radiation stress variations with time series of the wave group envelope and long-wave elevations, at different cross-shore locations: on the seaward (deeper) side of the step, at the step (but shifted very slightly on either side) and on the shoreward (shallower) side (Fig. 3). The radiation stress gradient over the flat bottom region is associated with the wave-group forcing. The sinusoidal radiation stress gradient is first negative, then positive as the wave group propagates within these flat regions (Figs. 3a,j), averaging to zero over time. At the step, the radiation stress gradient associated with the group is eclipsed by a stronger positive gradient of radiation stress (Figs. $3 \mathrm{~d}, \mathrm{~g}$ compared to Figs. 3a,j) resulting from the difference of amplitudes between the BLW on the seaward and shoreward sides as a result of the depth difference. In contrast to the flat bottom case, the radiation stress variation at the step is not zero on average. It is at this stage that both an iFLW and an oFLW are generated. Therefore, when the radiation stress gradient averages to zero over a wave-group period, only BLWs are generated; however, when the timeaveraged radiation stress becomes nonzero, FLWs are generated so that mass and momentum are conserved.

When inspecting the TLW signal on the seaward side, two negative signals separated in time are visible (Fig. 3c). The signal centered on $t^{*}=5.3$ represents the BLW propagating at the group and the one at $t^{*}=7.7$ is the oFLW. At the step (Figs. 3f,i), the TLW is composed of the BLW and the corresponding oFLW immediately on the seaward side, and of the BLW and the corresponding iFLW immediately on the shoreward side. On the shoreward side of the step (Fig. 3i), the iFLW and the BLW overlap, as they propagate in the same direction.

Last, to further verify the analytical model, we also consider results over a broader range of depth changes over the step. We specifically calculate the amplitudes of the FLWs for different nondimensional depth changes $\left(h^{*}=10 \times 10^{-3}\right.$ seaward and between $4 \times 10^{-3}$ and $10 \times 10^{-3}$ shoreward), both analytically [using Eqs. (17) and (18)] and numerically. The amplitudes of the FLWs for these cases are represented in Fig. 4, with the analytical solutions and the numerical predictions in very good 

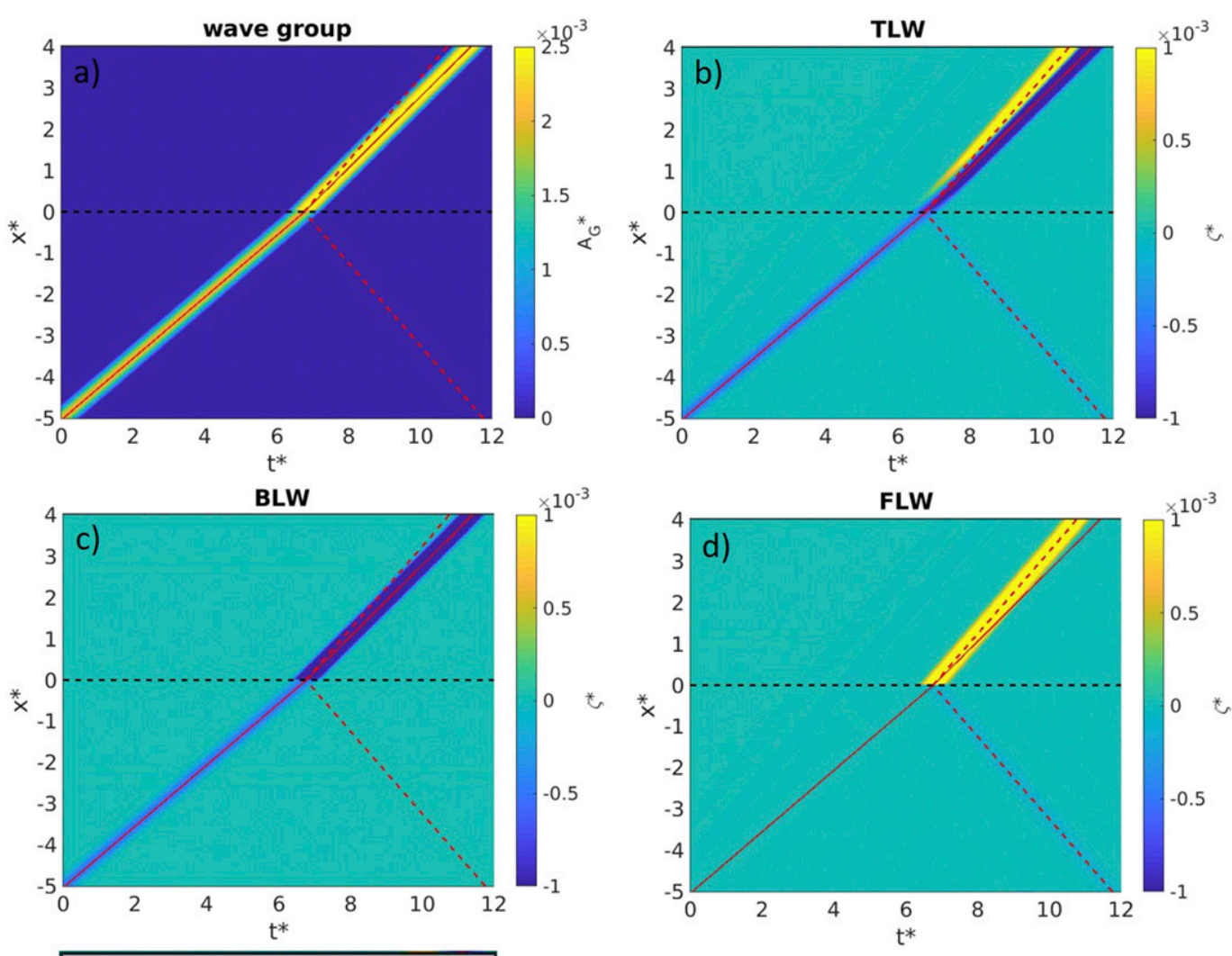

- - - propagation at free wave velocity propagation at group velocity

FIG. 2. Nondimensional (a) wave-group envelope; (b) TLW elevation, (c) BLW elevation, and (d) FLW elevation vs time and cross-shore direction. The dashed red lines represent the path of FLW (propagating at the linear shallow-water wave speed $\sqrt{g h}$, and the solid red lines represent the path of a BLW (propagating at the group velocity), generated at the step located at $x^{*}=0$ (indicated by the horizontal dashed line). For this example, $h^{*}=$ $10 \times 10^{-3}$ for $x^{*} \leq 0$, and $h^{*}=5 \times 10^{-3}$ for $x^{*}>0$.

agreement. These results emphasize that the analytical model can be used as a simple tool to assess the generation mechanisms of the long waves.

\section{b. Slope case}

The numerical model is next applied to the linear slope case, which in appendix $C$ we verify that the slope can be treated as a succession of discrete steps when the steps are sufficiently small. We obtain elevations and velocities for the TLW and the BLW elevation and then subtract the BLW from the TLW elevation to obtain the cFLW signal.

In Fig. 5, we represent the envelope of the wave group and the response of the TLW. The resulting TLW signal is composed of a positive part and a negative part, with the positive component of the TLW propagating ahead of the negative component (Fig. 5b). The signal from the BLW is negative and propagates at the group velocity. The cFLW signal is consistently positive (Fig. 5d). FLWs are generated successively at each depth variation as the group advances at $C_{g}$. The most recently generated FLW is larger than the previous one as it is generated in shallower water. Based on its local generation with the wave group, this can suggest that the cFLW propagates at $C_{g}$; however, it is a superposition of FLWs generated at different times along the path of the wave group that propagate at $\sqrt{g h}$. This is illustrated in Fig. $5 d$, where the paths of a few of the FLWs generated along the path of the wave group are represented (dashed red line crossing $x^{*}=0, t^{*}=0$ ).

The nondimensional radiation stress gradient versus time is represented in Fig. 6 (top row) at two cross-shore locations on the slope. As with the signal in the flat bottom region of the step case (Figs. 3a,d), the signal is sinusoidal (first negative and then positive). However, unlike over a flat bottom, the timeaveraged value is not zero $\left(\partial S_{x x}^{*} / \partial x^{*}=2 \times 10^{-5}\right.$ and $3 \times$ $10^{-5}$ at $x^{*}=0.5$ and $x^{*}=5.5$, respectively) and FLWs are generated that are superimposed on the BLW. The timeaveraged radiation stress difference increases as the depth decreases, and the amplitude of the FLW generated likewise increases.

The results also indicate a growing phase shift between the wave-group envelope and the TLW as the depth decreases (Figs. 6c,f). At $x^{*}=0.5$, where $h^{*}=15 \times 10^{-3}$, the amplitudes of the TLW and BLW are very similar, such that the TLW is still approximately in antiphase with the group (only $\sim 10^{\circ}$ behind the BLW). The cFLW is small and propagates well in front of the group (phase shift $\sim 70^{\circ}$ ). The cFLW is not symmetric around its 

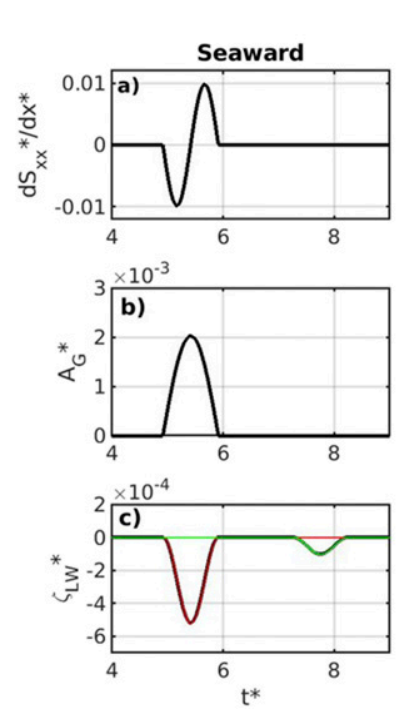
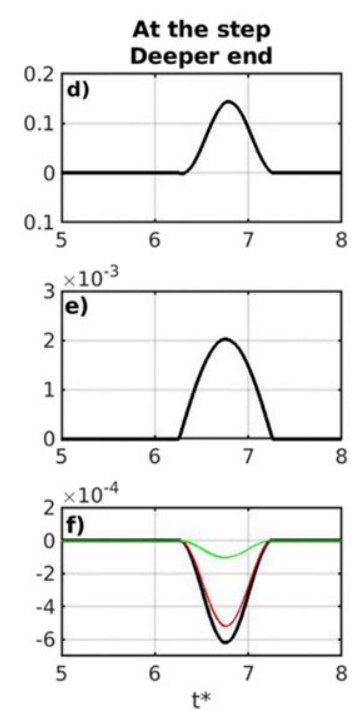
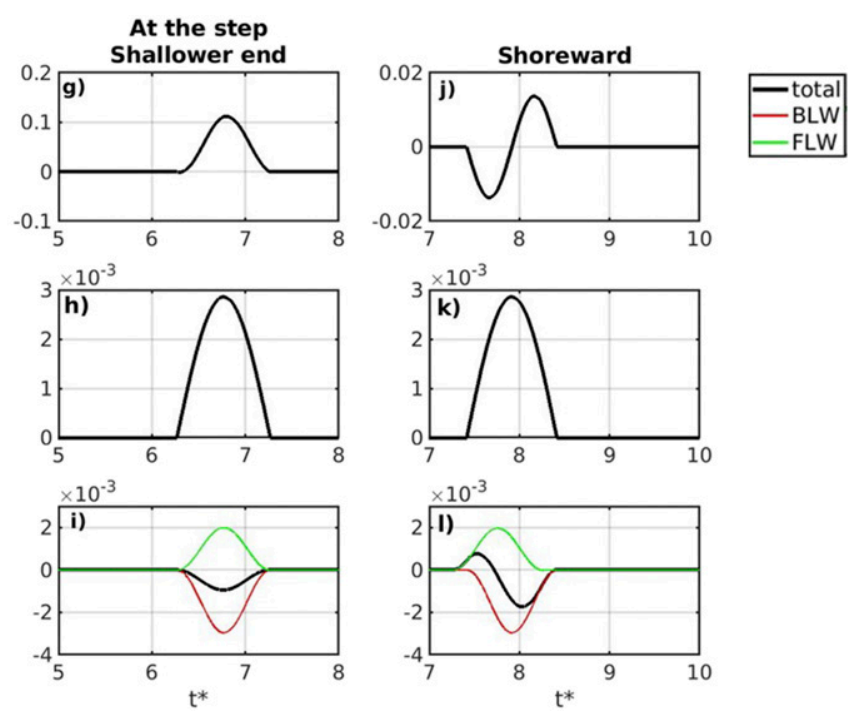

FIG. 3. Temporal evolution of (top) radiation stress gradient, (middle) wave-group envelope, and (bottom) long-wave elevation vs time at four different cross-shore locations: (a)-(c) on the seaward side, (d)-(f) at the step on the deeper seaward side, (g)-(i) at the step on the shallower side, and (j)-(l) on the shallower shoreward side.

peak because of the small FLWs generated offshore, which are older (generated earlier). These older FLWs propagate faster than the group and they gradually move out in front. They overlap with the locally generated FLW, but their peaks are shifted toward smaller $t^{*}$. At $x^{*}=5.5$, where $h^{*}=8 \times 10^{-3}$, the amplitude of the cFLW is almost equal to the amplitude of the BLW, and it is almost in antiphase with the BLW, such that the amplitude of the TLW is smaller than the amplitude of the BLW. The cFLW is still shifted in front of the group (phase shift $\sim 40^{\circ}$ ), so the phase between the TLW and the group shifts closer to $90^{\circ}\left(110^{\circ}\right.$, versus $170^{\circ}$ at $\left.x^{*}=0.5\right)$.

Using Figs. 5 and 6, we have illustrated how the total solution (the TLW) is a superposition of a BLW and a cFLW. Ignoring the small oFLWs, the generation of the cFLW signal is interpreted as follows. At the offshore end of the slope, a small FLW is initially generated. The BLW that is present is much larger than the FLW, so the amplitude of the TLW is almost equal to the amplitude of the BLW (Fig. 6c). At the next finite decrease in depth, a new FLW contribution is generated. This FLW is larger than the previous FLW and lags behind it, due to the preceding FLW propagating faster at the free wave velocity (as opposed to the group velocity associated with the short-wave group). This process occurs successively as the group propagates over the sloping bottom. Eventually, the amplitude of the FLW generated in shallow water is comparable to the amplitude of the BLW (Fig. 6f). Given that each FLW generated earlier propagates faster, yet are smaller in amplitude, the cFLW signal is not symmetric around its peak and has an extended tail on the shoreward side (toward smaller $\left.t^{*}\right)$ (Fig. 6c, green line).

We now compare the analytical model with the numerical results. We calculate the phase lags and the amplitude of the long waves from the analytical model [Eqs. (19) and (20)], and the phase lags and the amplitude of the long waves from the numerical model, by cross-correlating the long-wave elevation and the group envelope and identifying the maxima and minima of elevation (Fig. 7). We find close agreement between the numerical model and the analytical solution, thus providing further support that the analytical model captures the dominant mechanisms responsible for the FLW generation. Note that while Fig. 7 focuses on the long-wave response for a particular slope and wave condition as an illustrative example, in appendix B we also consider a range of $\beta$ and wave-group frequencies and find the analytical model to be similarly robust. This also further reinforces that the assumptions made in the analytical model are reasonable, including neglecting the generation of an oFLW within the analytical solution.

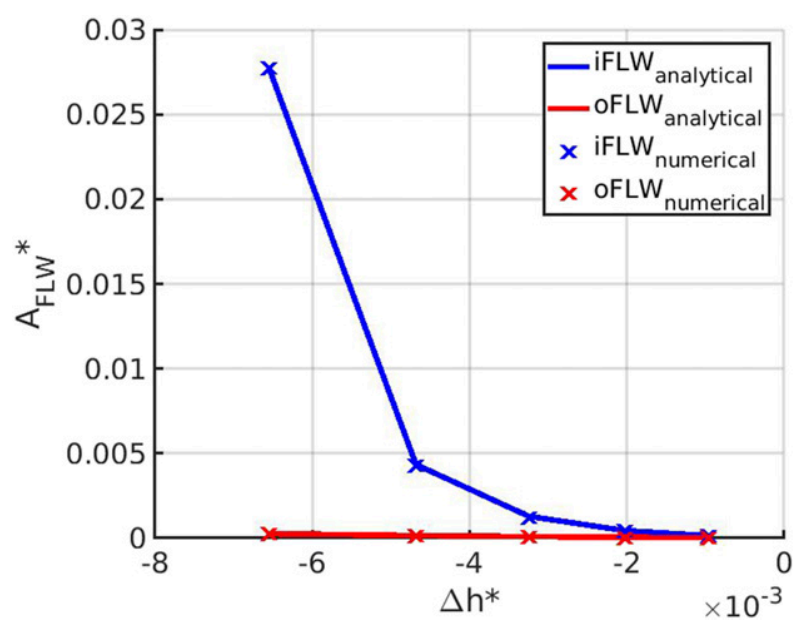

FIG. 4. Amplitude of the iFLW and oFLW as a function of step depth variations (seaward depth $h^{*}=15 \times 10^{-3}$ and varying onshore depth, where $\Delta h^{*}$ is the depth difference), comparing the numerical solutions (crosses) and analytical solutions (solid lines). 

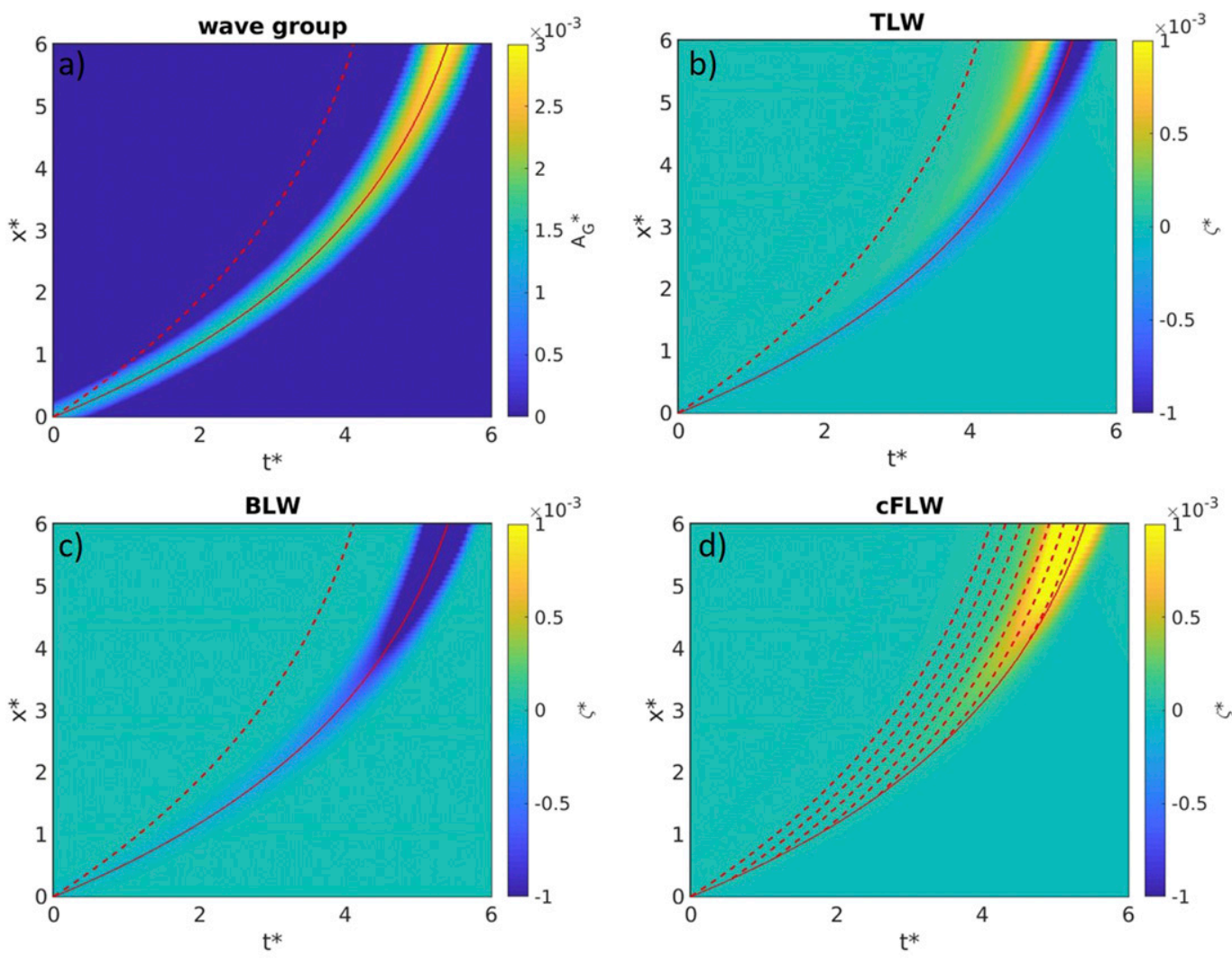

- - propagation at free wave velocity

FIG. 5. Nondimensional (a) wave-group envelope, (b) TLW elevation, (c) BLW elevation, and (d) cFLW elevation vs time and cross-shore direction. The solid red lines represent the path of a BLW (propagating at the group velocity) and the dashed red lines represent the path of FLW (propagating at the shallow-water wave speed $\sqrt{g h}$, generated at $x^{*}=0$ ). In addition, in (d) the path of a few FLWs, generated along the path of the wave group are represented in dashed red lines (in reality, FLWs generated at each depth variation, but only a small number of them are represented here).

\section{Comparison with J03}

Our approach assumes that at each location the total longwave solution is a combination of a particular solution, the (forced) BLW and homogenous solutions, the FLWs. This approach is different to that of J03, and other studies (Guérin et al. 2019; Zhang et al. 2020), who calculate one unique solution, referred to as shoaling BLW. We compare our total solution (TLW) to the shoaling BLW solution and find a very good agreement in terms of phase relationship and shoaling exponent (Fig. 8) with J03's solution, who used a perturbation approach and with exact solutions obtained by variation of parameters.

In Fig. 8, we also represent the phase shift and the shoaling exponent for the BLW and FLW components. For $k_{f} h<0.4$, the total shoaling exponent is almost equal to the BLW shoaling exponent, since BLW are dominant over FLWs. As the depth decreases, the shoaling exponent of the TLW decreases, becoming smaller than the shoaling exponent of the BLW, as the FLWs are not negligible anymore. The addition of
FLWs, in antiphase to the BLW, lowers the total amplitude. This shows how the growth of shoaling BLW is limited by the presence of FLWs.

\section{Validation: Comparison with experimental data and phase-resolving wave model output}

We have confirmed that the analytical model can reproduce the long-wave response predicted by the linear numerical model to describe the generation of FLWs by nonbreaking wave groups. Given that nonlinear dynamics have the potential to influence the results, which would not be captured by the linearized model, we further assess the solutions against experimental data. In this section, we validate our analytical solutions against measurements from laboratory experiments (van Noorloos 2003), which consisted of bichromatic wave groups shoaling on a plane beach, and with numerical simulations of the same van Noorloos (2003) experiment using a nonhydrostatic wave-flow model based on the Reynoldsaveraged Navier-Stokes equations. 

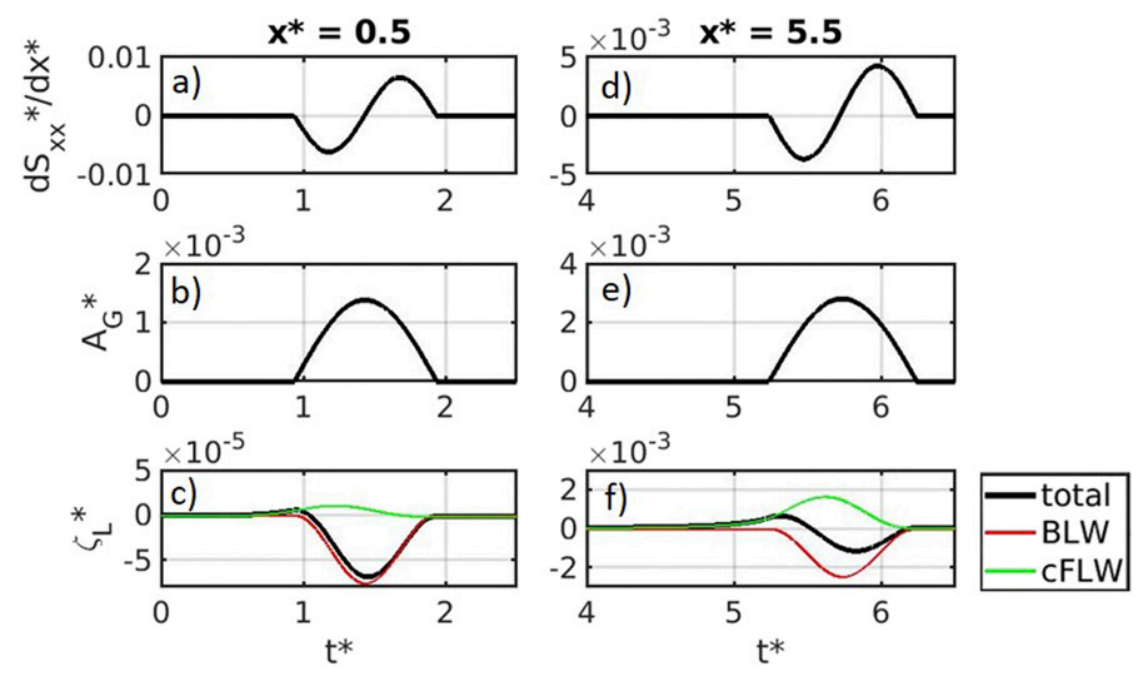

FIG. 6. Temporal evolution of (a),(d) radiation stress gradient, (b),(e) wave-group envelope, and (c),(f) long-wave elevation at two different cross-shore locations: (left) $x^{*}=0.5\left(h^{*}=15 \times\right.$ $\left.10^{-3}\right)$ and (right) $x^{*}=5.5\left(h^{*}=8 \times 10^{-3}\right)$.

\section{a. Experimental measurements}

The van Noorloos (2003) dataset is used to compare the evolution of the incoming long waves over a sloping bed prior to breaking, which are compared to the analytical model. In the van Noorloos (2003) experiment, nine bichromatic cases were tested, where the amplitude of the wave-group envelope varied between 0.022 and $0.072 \mathrm{~m}$ and the group frequency varied between 0.0977 and $0.1953 \mathrm{~Hz}$, which were generated by a wave maker with active reflection compensation. The plane slope was 1:35 and the maximum offshore depth was $0.7 \mathrm{~m}$. The wave gauges were regularly spaced every $0.5 \mathrm{~m}$ seaward of the surf zone and were reduced to every $0.3 \mathrm{~m}$ within the surf zone.

\section{b. Phase-resolving wave model simulations}

SWASH is a nonhydrostatic wave-flow model that numerically solves the incompressible Reynolds-averaged NavierStokes equations (Zijlema et al. 2011). In recent years, nonhydrostatic models such as SWASH have proven to be powerful tools to resolve the nonlinear evolution of waves in coastal regions at both laboratory and field scales (GarcíaMedina et al. 2017; Gomes et al. 2016; Smit et al. 2013), including the nearshore dynamics of infragravity waves (Rijnsdorp et al. 2014, 2015; de Bakker et al. 2016). Given its capabilities in resolving such nonlinear wave dynamics, several recent studies have adopted this modeling framework to further explore nearshore infragravity-wave processes (de Bakker et al. 2016; Mendes et al. 2018; Ruju et al. 2019).

We apply SWASH with forcing that matches the bichromatic wave conditions in the van Noorloos (2003) experiments. The model was run with two vertical layers, with default values for bottom friction dissipation and breaking. To mimic the active absorption of the laboratory wavemaker, a weakly reflective wavemaker was used in the model simulations to generate incoming waves and absorb reflected waves (Zijlema et al. 2011). A second-order boundary condition was used to properly generate incoming bound waves and suppress the generation of spurious free infragravity waves (Rijnsdorp et al. 2014). The settings were chosen based on Rijnsdorp et al. (2014), who validated SWASH against the van Noorloos (2003) experiment.

\section{c. Comparisons with the analytical model}

We investigate how the analytical model compares with the laboratory experiments and SWASH modeling results, in terms of free and forced components of the long wave. In this section, we present the results of the comparison for two selected cases, A2 and B4 (Table 2). However, the results for the other cases are presented in appendix D.

The experimental data and SWASH output are processed in the same way. We filter elevation measurements using a cutoff frequency of $0.25 \mathrm{~Hz}$, chosen between the short-wave frequencies $\left(f_{1}\right.$ and $\left.f_{2}\right)$ and wave-group frequency $(f)$, in order to
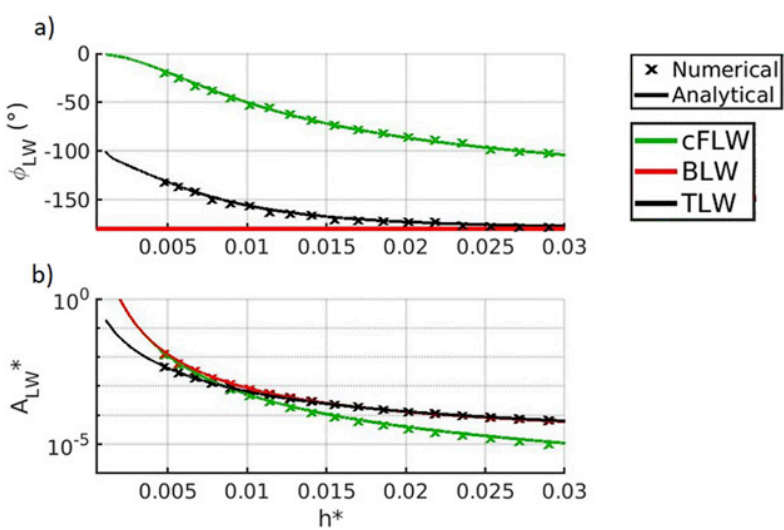

FIG. 7. (a) Phase lags between the wave-group envelope and the different long-wave contributions and (b) amplitude of the long wave, calculated numerically and analytically. 

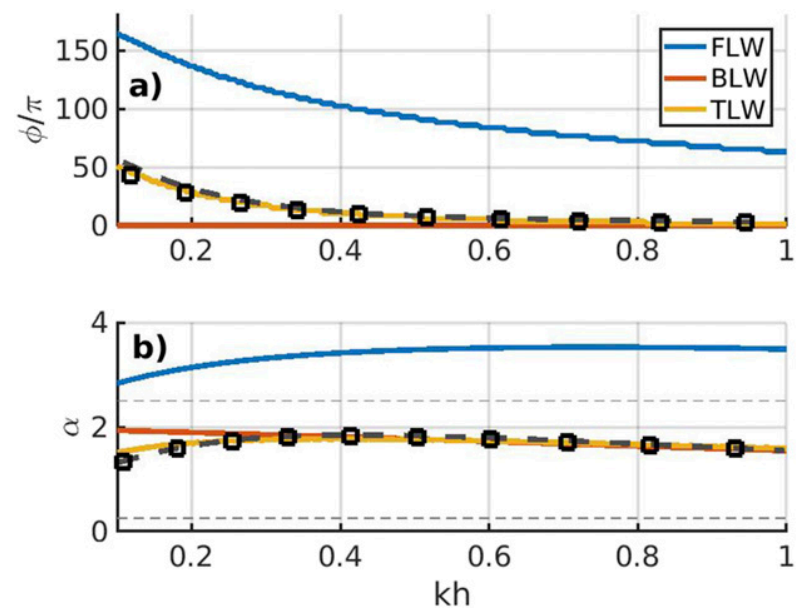

FIG. 8. (a) Phase lags between the wave-group envelope and the different long-wave contributions and (b) the shoaling exponent $(\alpha)$. Solid lines: analytical results, squares: J03's analytical solution, amplitude evolution response (AER), dashed lines: J03's numerical solution, exact linear response (ELR) solution, dashed horizontal gray lines represent $\alpha$ according to Green's law ( $\alpha=0.25)$ and for the shallow-water equilibrium BLW solution $(\alpha=2.5)$.

separate the low-frequency (LF) and the high-frequency (HF) signals. We further decompose the LF signal into iFLW, oFLW, and BLW as follows. The amplitude of the BLW is computed from the measured wave-group amplitude using Eqs. (3) and (7). Once the BLW is removed from the LF signal, we obtain the total FLW signal. We then decompose the FLW elevation into its iFLW and oFLW contributions following the method described in appendix E.

We next apply Eqs. (19)-(21) to calculate the amplitude and phase of the long waves from the wave-group envelope and local depth. We calculate the wave-group envelope as the lowpass-filtered (with cutoff frequency $0.25 \mathrm{~Hz}$ ) absolute value of the Hilbert transform of the high-frequency (short-wave) elevation. The wave-group envelope (Fig. 9) is used to first calculate the amplitude and phase of the BLW and, from these, the amplitudes of the FLW and TLW.

We decompose the TLW from all datasets (analytical model, experimental observations, and SWASH model results) into its free incoming (iFLW), free outgoing (oFLW) and bound (BWL) components (as described previously), and we present the values of the correlation function between the envelope of the short-wave group and the elevation of the long waves in Fig. 10. The BLW is strongly correlated with the wave group envelope and the time lag associated with the minimum negative correlation is zero for all cases, which is expected because the BLW elevation is calculated from the wave-group envelope [Eqs. (3) and (7)]. For the TLW, the time lag associated with the negative correlation (white dash-dot line) is close to zero at the bottom of the slope and shifts slightly away from zero as waves propagate over the slope, until breaking occurs at about $x=23 \mathrm{~m}$. FLWs are already present before the start of the slope (i.e., before $x=7.5 \mathrm{~m}$ ), which indicates the presence of
TABLE 2. Amplitudes and frequencies of the short waves in the van Noorloos (2003) bichromatic wave experiments.

\begin{tabular}{lcccccc}
\hline \hline Case & $a_{1}(\mathrm{~m})$ & $a_{2}(\mathrm{~m})$ & $f_{1}(\mathrm{~Hz})$ & $f_{2}(\mathrm{~Hz})$ & $f(\mathrm{~Hz})$ & $\beta /(2 \pi f)$ \\
\hline A2 & 0.06 & 0.012 & 0.6470 & 0.5005 & 0.1465 & 0.0310 \\
B4 & 0.06 & 0.030 & 0.6470 & 0.5005 & 0.1465 & 0.0310 \\
\hline
\end{tabular}

spurious FLWs generated at the wave maker, as no FLWs are otherwise expected on the flat bottom section. However, at approximately $x=15 \mathrm{~m}$, the FLWs generated along the slope are large enough to dominate over the signal from the spurious waves. From $x=15 \mathrm{~m}$ to the breakpoint, the maximum correlation between the envelope and the FLW shifts closer toward zero, causing the shift in time lag observed in the TLW compared to the BLW. While we are not studying the effect of breaking, we note that in case A2, the correlation between the BLWs and the envelope is much smaller after breaking, particularly with the SWASH run (Figs. 10b,e) while the correlation is well conserved in case B4, indicating that wave groups are likely destroyed in case A2 but substantial groupiness remains in case B4. When groupiness remains, the time lag between the envelope and the FLW changes and becomes zero, possibly indicating breaking of the FLW, and generation of new FLWs after breaking, as the depth keeps decreasing.

Based on the cross-correlation time lags, we calculate the phase lags between the wave-group envelope and the long waves. We also calculate the amplitude of the long waves as the mean difference between the elevation maxima and minima divided by two. Comparisons between the laboratory experiment, the SWASH runs and the analytical model are presented in Fig. 11. The analytical model, forced with the measured wave-group envelope (Fig. 9), generally reproduces the amplitude and phase lag of the iFLW and TLW (Fig. 11) measured in the experiment very well. We note, however, that for the specific iFLW component, the phase lag is not well predicted in deeper water $(x<12 \mathrm{~m}$ which corresponds to $h>0.5 \mathrm{~m})$ and the measured phase lag tends toward $180^{\circ}$ (i.e., in antiphase with the wave group) as the depth increases, which may again indicate the presence of some spurious FLW energy that is in phase with the group before the start of the slope, i.e., at the boundary. This may happen at the wavemaker boundary for both the experiments and the SWASH model if the boundary is not fully absorbing. However, it is important to emphasize that the iFLW signal is extremely small in this deep region, which adds large uncertainty to the phase calculations for the FLW. Any perturbation of the short-wave group may lead to the generation of FLW, which can alter the phase relationship for these extremely small FLWs. As depth decreases, the FLWs rapidly become larger and are therefore much less affected by noise. The oFLWs remain small in both the measurements and SWASH, which further supports neglecting them in the analytical model. We note that the short waves in this experiment are nonlinear second-order Stokes waves (Le Mehauté 1976), for $x<23 \mathrm{~m}$. While we do not expect the linear model to reproduce nonlinear effects, the results presented in Fig. 11 show that the linear model reproduces the trends observed in the 

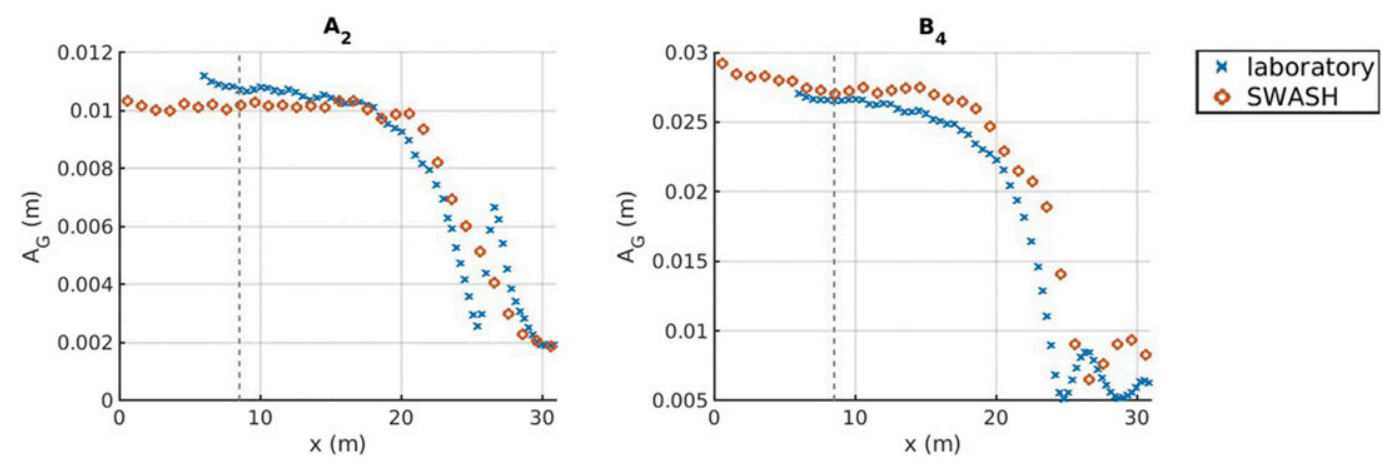

FIG. 9. Amplitude of the wave-group envelope, vs cross-shore position, for two cases: (a) A2 and (b) B4. The dashed vertical gray line indicates the start of the slope.

laboratory experiment and SWASH runs with remarkable agreement with waves nonlinear at the second order.

\section{Discussion}

This study investigated the decomposition of total longwave motions in the nearshore zone into free and forced components by use of the linearized shallow-water equations at the short-wave group scale with radiation stress forcing. The total solution we propose, the TLW, is equivalent to the shoaling BLW solution that has been previously described (J03; Guérin et al. 2019; Zhang et al. 2020). However, by decomposing the TLW into free and forced components, the present approach provides insight into the physical processes responsible for the TLW signal. This approach provides additional insight into a mechanism generating FLWs when nonbreaking short-wave groups propagate over changing bathymetry (Mei and Benmoussa 1984; Nielsen 2017; Moura and Baldock 2019) and it explains why the TLW growth is less than the wave growth of a (locked) BLW and more than that of a shoaling FLW, and why the phase between TLWs and the short-wave groups shifts as groups propagate over a slope. In particular, when wave groups enter shallow water, the BLWs are expected to approach a resonance condition based on Eq. (7), when the group velocity tends to $\sqrt{g h}$ and the denominator approaches zero. However, our study shows that a FLW of the same amplitude and opposite phase adds to the BLW signal, which prevents the TLW amplitude from increasing dramatically.

\section{a. FLW generation}

The FLWs generated through the depth-variation mechanism propagate independently from the short-wave group, and this mechanism may be compared to other mechanisms known to generate FLWs in the nearshore by short-wave breaking, i.e., breakpoint forcing (Symonds et al. 1982) and bound-wave release (Masselink 1995). We compare schematically the response of FLWs to generation from depth variation, breakpoint forcing and bound-wave release in Fig. 12. The FLWs generated through depth variation and breakpoint forcing are expected to be in phase with each other, while the shoreward propagating FLW generated through bound-wave release should be in antiphase with them. The amplitude of the breakpoint-forced FLWs will be enhanced by the FLWs generated through depth variations. In contrast, the amplitude of released bound waves will be diminished by the presence of FLWs generated through depth variations.

Radiation stress gradients are important to the generation of FLWs, given that they provide the source term in the momentum conservation equation [Eq. (2)]. When the average value of the radiation stress gradient over a wave-group period is zero, the system is in equilibrium and only BLWs are generated. However, when the average value is not zero, FLWs are generated. This is well known to be the case within the surf zone when the amplitude of the wave-group envelope changes during short-wave breaking, which leads to breakpoint forcing (Symonds et al. 1982). However, it is also the case when nonbreaking waves encounter depth changes, which is the mechanism we focus on here. This highlights similarities between the single step case and breakpoint forcing, as in both cases, both an iFLW and an oFLW are generated.

Although the primary motivation for introducing the step case was to apply it successively to understand mild slope cases, the mechanism of FLWs generation in response to depth changes is also in place when the depth changes abruptly, such as the steep slopes of coral reefs, where strong infragravity wave signals have been measured (Pomeroy et al. 2012; Péquignet et al. 2014; Beetham and Kench 2011; Buckley et al. 2015; Masselink et al. 2019). In the case of a step, an iFLW is generated where the depth changes and propagates toward the shore, ahead of the BLW. At the same time, an oFLW is generated and propagates seaward. This oFLW is smaller than the iFLW but not negligible, on a steep slope, and is in antiphase with the group (not taking the propagating time lag into account). This same pattern is usually considered as the signature of the breakpoint forcing mechanism (Symonds et al. 1982) and thus it raises questions about how to identify and distinguish these two long-wave generation mechanisms in the case of a very steep slope. For instance, a strong breakpoint forcing signal is usually described on fringing reefs (Pomeroy et al. 2012); in such cases the depth variation is very steep, which would cause strong radiation stress gradients, and thus would also be expected to generate large FLWs propagating both shoreward and seaward through the mechanism described in this paper, although further investigation will be needed to estimate the amplitude and phase relationships of the FLWs generated. 
TLW
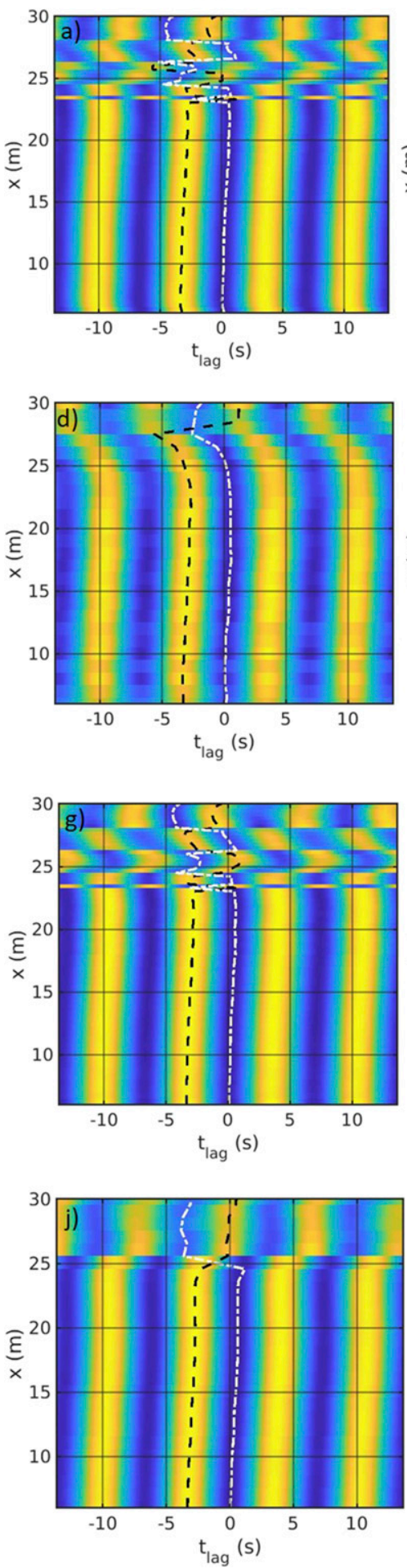

BLW
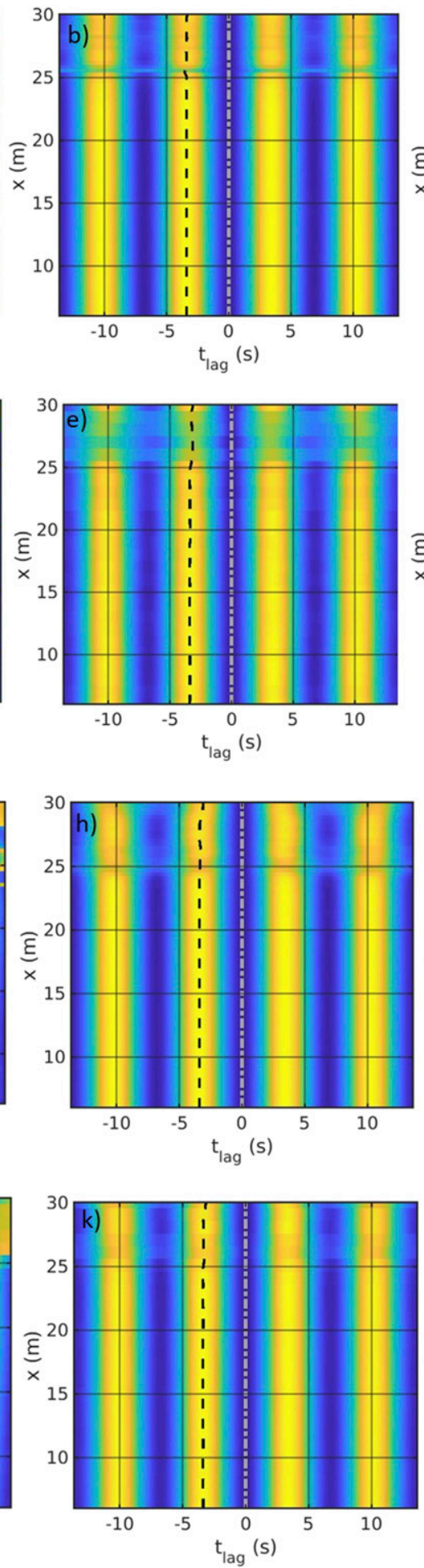

FLW
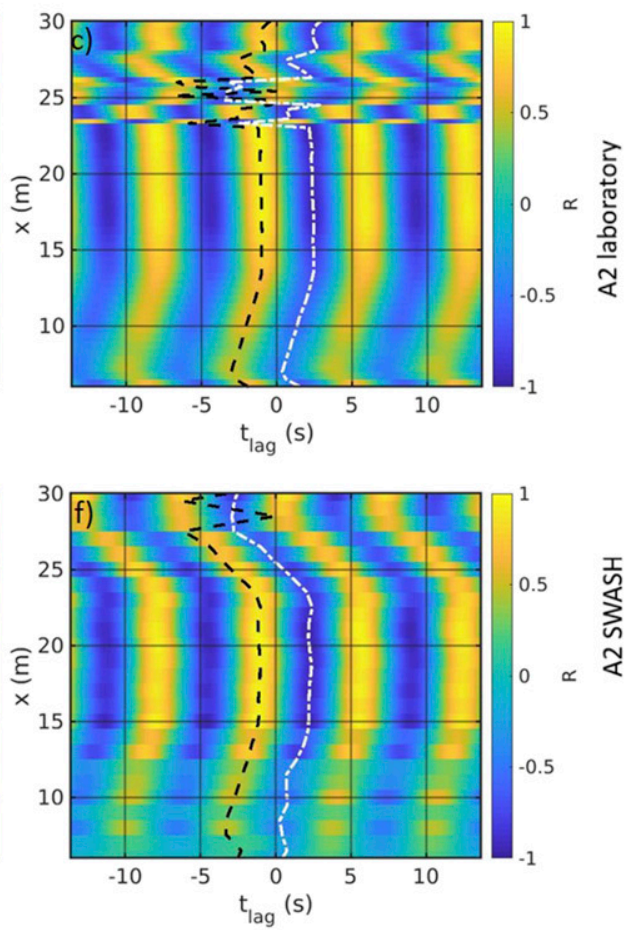

FIG. 10. Correlation functions $(R)$ between the wave-group envelope and long waves for (a)-(f) A2 case and (g)-(l) B4 case showing laboratory results in (a)-(c) and (g)-(i) and SWASH run results in (d)-(f) and (j)-(l): (left) TLW, (center) BLW, and (right) FLW. The dashed black line indicates a local maximum correlation, and the dash-dot white line indicates a local minimum correlation. 

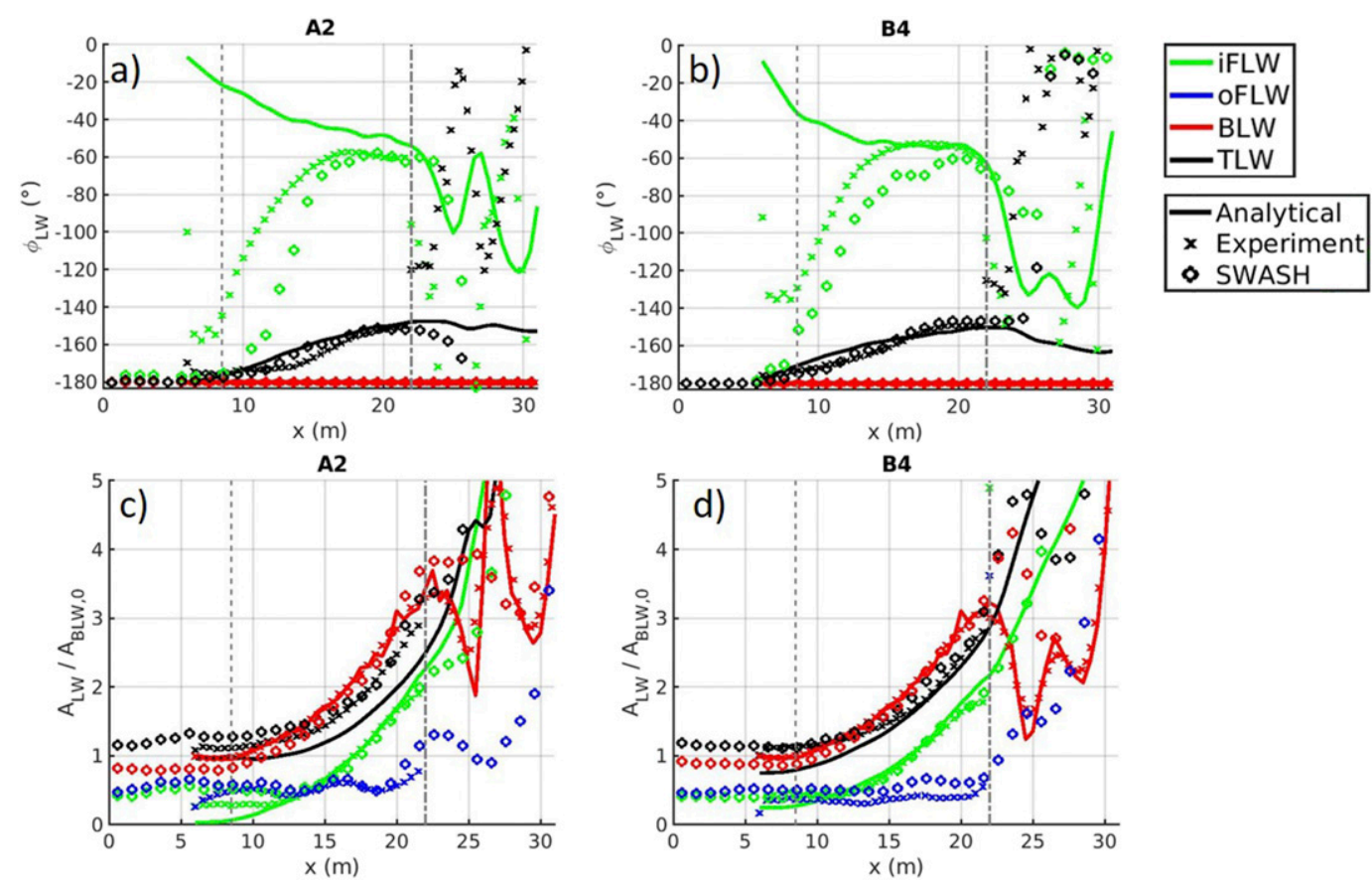

FIG. 11. Phase lag between long wave and wave-group envelope for (a) case A2 and (b) case B4, and long-wave amplitude, relative to the BLW amplitude at the start of the slope, for (c) case A2 and (d) case B4. Note that the vertical dashed gray line indicates the start of the slope, and the dash-dot gray vertical line indicates the approximate location of the short-wave breakpoint. Data are smoothed using a moving average over $3 \mathrm{~m}$.

\section{b. Phase shift and growth rate}

The linearized models (numerical and analytical) were found to reproduce the dominant trends in both an experimental dataset and output from a fully nonlinear phase-resolving wave model. Importantly, through use of the approach detailed here, we were able to clarify the dominant physical processes responsible for the evolution of long waves in the nearshore. As a wave group propagates into shallower water, each finite depth variation causes the generation of two FLWs, one incoming and one outgoing. The iFLW is in phase with the group envelope, while the oFLW is in antiphase. The oFLW is small compared to the iFLW and propagates away from the group, so its contribution to the TLW is negligible. The iFLW propagates in the same direction as the group, but faster than the group. As iFLWs are successively generated, they lag each other. These FLW components overlap to create a cFLW, where the smaller iFLWs propagate ahead of larger iFLWs. The TLW is then a superposition of a BLW and a cFLW. The amplitude and phase relationships of the TLW depend on the relative amplitude (relative to depth and group frequency) of the depth variation, as described below and summarized in Table 3. For the purpose of discussion, Zhang et al. (2020) investigated the effect on the phase relationship of the addition of a FLW to the BLW, in antiphase with the wave group. They suggested that the FLW is likely to shift the phase between the long wave and the wave group. However, in their example, the origin of the FLW is not specified and is added (at the toe of the slope) to the shoaling BLW (TLW), while in this present study we consider the addition of a FLW generated from depth variation to the (locked) BLW. Moura and Baldock
(2017) also noted that adding FLWs to the BLW shoaling process could alter the phase relationship between long waves and wave groups.

While the evolution of long waves has been considered analytically/numerically in a number of prior studies (J03; List 1992; Battjes et al. 2004; Zhang et al. 2020) these prior studies have not explained all of the relevant physics, as there was no distinction between BLW and FLW components. Indeed, Battjes et al. (2004) had pointed out that the amplitude of BLW in shallow water was "less than it would be if the equilibrium theory of Longuet-Higgins and Stewart (1962) were applied over a sloping bed" and Okihiro et al. (1992) acknowledged that the shallow water limit of applicability for bound wave theory is not well understood. Here we found that the combination of those two waves of almost the same amplitude and almost in antiphase keeps the growth rate of the TLW much milder than it would be in the absence of FLWs. This helps explain why the theoretical prediction of a dramatic increase of long-wave amplitude for BLWs as they enter resonance with $C_{g}$ tending to $\sqrt{g h}$ [Eq. (7)] is not realized, due to the presence of FLWs.

\section{c. Propagation of FLWs}

If the slope is followed by a flat region and the generation of FLWs consequently ceases, the FLWs propagate faster than the BLW, implying that the BLW and the latest generated FLW are no longer in antiphase. Li et al. (2020) recognized the effect of wave history on the long-wave amplitude, as they identified an increase of amplitude over the flat portion of a shoal. Following our reasoning, we can help explain the observation made by 


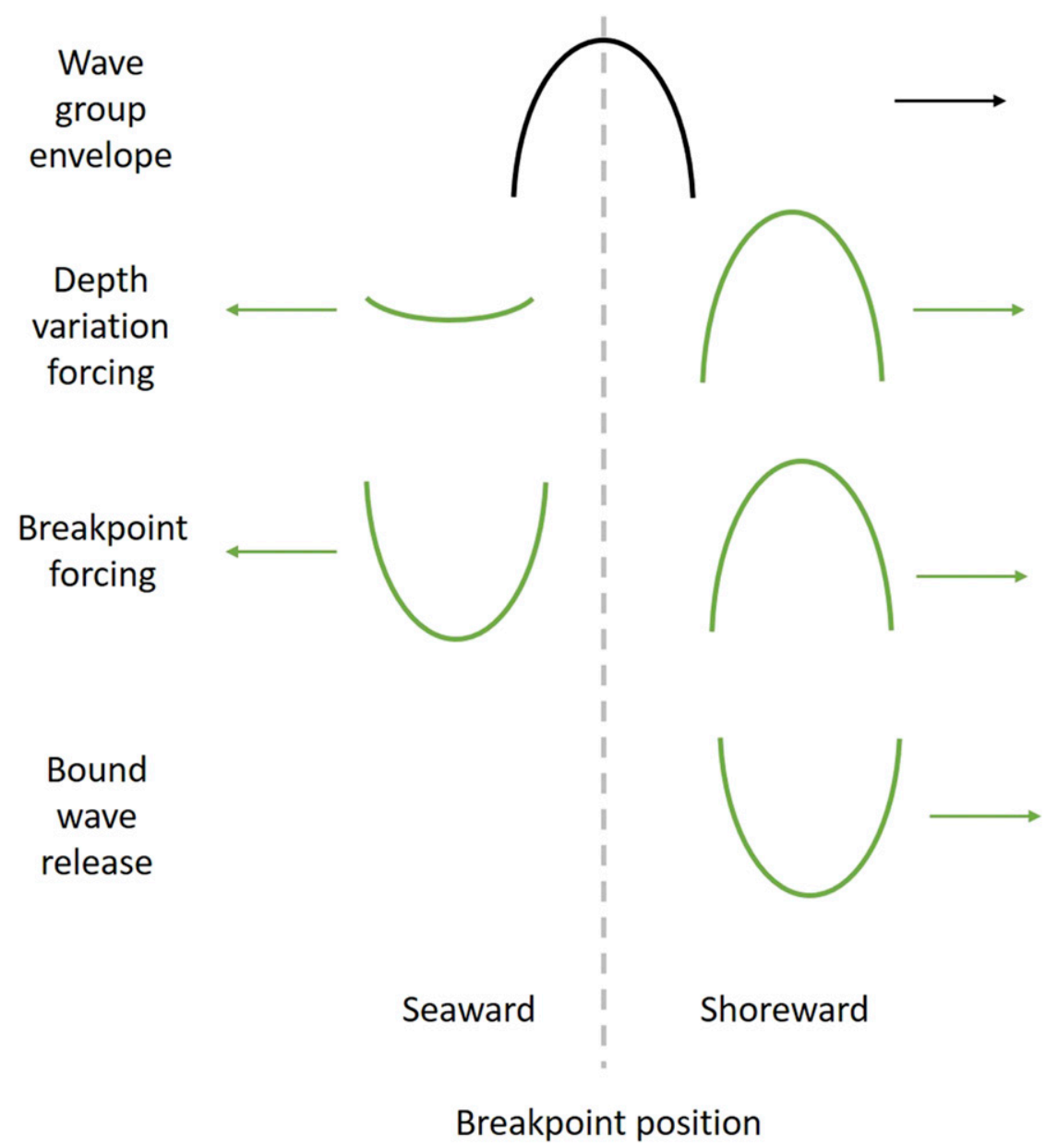

FIG. 12. Schematic representation of the FLW crests and troughs, associated with the crest of a wave-group envelope.

Li et al. (2020). We run our numerical model with the conditions described in Li et al. (2020), except the length of the plateau is extended to $1200 \mathrm{~m}$ instead of $20 \mathrm{~m}$ (Fig. 13). As the wave group reaches the end of the slope and the start of the plateau, a large FLW is generated. At that point, the crest of the FLW is aligned with the trough of the BLW (they are locally in antiphase), so the amplitude of the TLW is relatively small (Fig. 13d, $x=75 \mathrm{~m}$ ). In the case of continuous forcing, as the BLW and the FLW propagate over the flat portion of the shoal, their respective amplitudes do not vary, but the FLW propagates faster than the BLW, so the crest and the trough are no longer aligned (the phase lag between them is different from $180^{\circ}$ ), and the amplitude of the TLW increases (reaching a maximum at $x \approx 300 \mathrm{~m}$ ). This is similar to a bichromatic wave group composed of two waves of different frequencies. At locations where the crest of one wave is aligned with the trough of the other wave, the amplitude of the group is minimum (Figs. 13d,h, $x=450 \mathrm{~m}$ ), and when both crests are aligned, the amplitude of the group is maximum (Figs. 13d,h, $x=250 \mathrm{~m}$ and $x=650 \mathrm{~m}$ ). Here

TABLE 3. Characteristics of the long wave for small and large relative depth variations.

\begin{tabular}{llcl}
\hline \hline & \multicolumn{1}{c}{ Depth variation } \\
\cline { 2 - 4 } & $\begin{array}{c}\text { No depth variation } \\
\text { (flat bottom) }\end{array}$ & $\begin{array}{c}\text { Small relative } \\
\text { depth variations } \\
\text { (i.e., deep end) }\end{array}$ & $\begin{array}{c}\text { Large relative depth variations } \\
\text { (i.e., shallow end) }\end{array}$ \\
\hline Characteristic of FLW signal & No generation of FLW & cFLW asymmetrical & $\begin{array}{c}\text { Locally generated iFLW } \gg \text { iFLW previously } \\
\text { generated in deeper water } \\
\text { cFLW } \approx \text { iFLW }\end{array}$ \\
$\begin{array}{l}\text { Amplitude and phase relationship } \\
\text { (relative to group) }\end{array}$ & $\begin{array}{l}A_{\mathrm{iFLW}}=0 \\
\varphi_{\mathrm{TLW}}=\varphi_{\mathrm{BLW}}=180^{\circ}\end{array}$ & $\begin{array}{l}A_{\mathrm{iFLW}} \ll A_{\mathrm{BLW}} \\
\varphi_{\mathrm{TLW}} \approx \varphi_{\mathrm{BLW}} \approx 180^{\circ}\end{array}$ & $\begin{array}{l}A_{\mathrm{iFLW}} \approx A_{\mathrm{BLW}} \text { (in antiphase) } \\
\varphi_{\mathrm{TLW}} \approx 90^{\circ}\end{array}$ \\
\hline
\end{tabular}



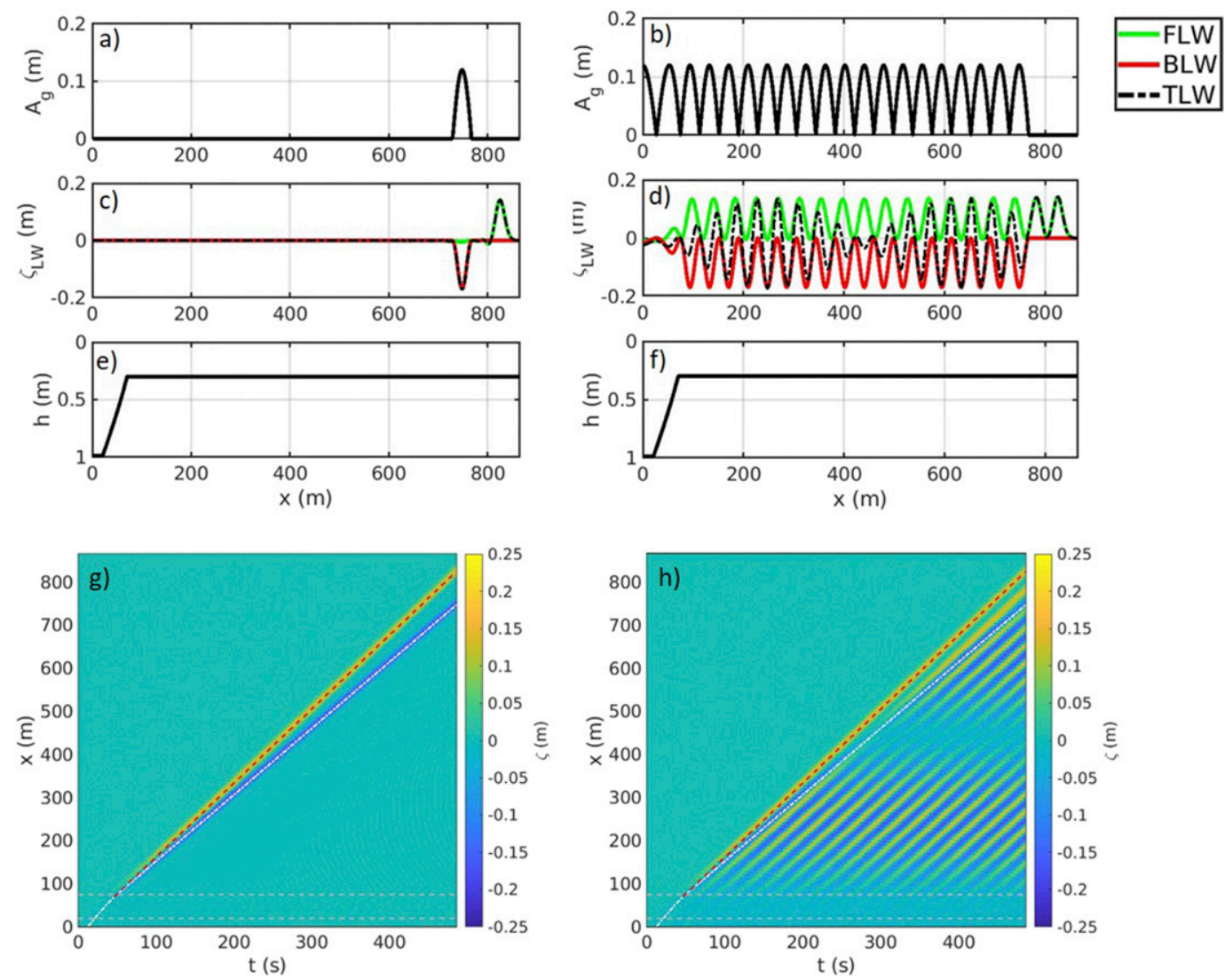

FIG. 13. (a),(b) Wave-group envelope; (c),(d) long-wave elevation, after a wave group has propagated over a flat bottom ( $0.55-\mathrm{m}$ depth), followed by a slope $(\beta=1 / 80)$, then a shallower flat bottom $(h=0.3 \mathrm{~m})$; (e),(f) bathymetry profile; and $(\mathrm{g}),(\mathrm{h})$ TLW elevation vs time and cross-shore direction. The dash-dot white lines represent the path of a BLW (propagating at the group velocity), and the dashed red lines represent the path of FLW (propagating at the shallow water wave speed $\sqrt{g h}$, generated at the end of the slope) Dashed gray horizontal lines indicate the start and the end of the slope. (left) Forcing with a single group and (right) forcing with a continuous group.

the frequencies of both waves are the same, so the group (supergroup) only exists in the spatial domain and the supergroup wavenumber is the difference between the wavenumber of the BLW and the wavenumber of the FLW. Here the wavelength of the supergroup is about $500 \mathrm{~m}$, so with a plateau only $20 \mathrm{~m}$ long, $\mathrm{Li}$ et al. (2020) are only seeing a very small portion of the supergroup. This example is interesting because this cannot be predicted using an analytical model, such as J03's, which does not identify FLWs. This phenomenon may have been observed in the field by Bertin et al. (2020) who interpreted it as the "merging" of a BLW and a FLW, propagating at different velocities until reaching shallow water where their respective velocities were equal.

This example with a long, shallow plateau after a slope also allows us to show that the transformation of BLWs involves the generation of FLWs as they are still present past the end of the slope and propagating away from the group, as illustrated in Figs. $13 \mathrm{~d}$ and $13 \mathrm{~h}$, where the FLWs are ahead of the group by two wavelengths. By forcing the model with a single group rather than a continuous group, we observe the independent propagation of the FLWs away from the group past the end of the slope (Figs. 13c,d, $x>750 \mathrm{~m}$ ). This confirms the physical presence of the FLWs that we predicted.

\section{d. Shoaling of long waves}

In the case of shoaling free waves only (no forced waves), the variation of the wave amplitude can be determined from the long-wave energy balance:

$$
\frac{\partial E}{\partial t}+\rho g h \frac{\partial U_{\mathrm{FLW}} \zeta_{\mathrm{FLW}}}{\partial x}=0,
$$

where $E$ is the long-wave energy. For harmonic waves, this leads to

$$
\frac{\partial}{\partial x}\left(C_{g}\left\langle E_{\mathrm{FLW}}\right\rangle\right)=0
$$

where the angle brackets represent time average over a longwave period. The energy flux $F=c_{g} E_{\mathrm{FLW}}$, averaged over a long-wave period, is constant. In the case a wave propagating over a depth variation: $E_{2} C_{g, 2}=E_{1} C_{g, 1}$, where $E_{1}$ and $C_{g, 1}$ are the energy and group velocity of the wave on one side and $E_{2}$ and $C_{g, 2}$ the amplitude and group velocity on the other side. Assuming a mild slope, the reflected signal may be ignored (Svendsen and Hansen 1977; Le Mehauté 1976), which leads to $A_{2} \sqrt{C_{g, 2}}=A_{1} \sqrt{C_{g, 1}}$, where $A_{1}$ is the amplitude of the wave on one side and $A_{2}$ the amplitude on the other side. 

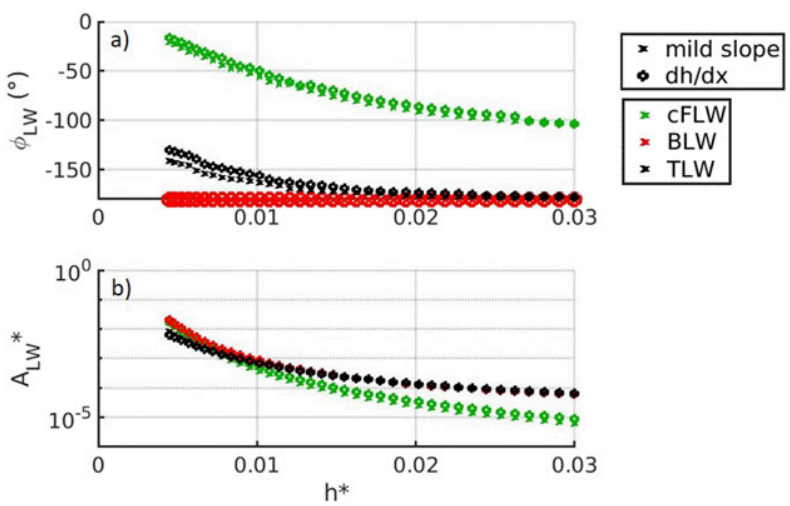

FIG. 14. (a) Phase lags between the wave-group envelope and the different long-wave contributions and (b) amplitude of the long wave, calculated numerically, with and without the mild-slope approximation.

However, in the case of BLWs, the amplitude is imposed by the wave group, so the amplitude of the BLWs cannot be adjusted to conserve the energy as depth changes. FLWs are generated to allow the conservation of energy in the system as short-wave groups propagate over varying depth. It is therefore consistent to relate the present approach to "shoaling of BLWs," as done in literature (Battjes et al. 2004; J03; Zhang et al. 2020; van Dongeren et al. 2007), to describe the phenomenon by which FLWs are generated along BLWs as shortwave groups propagate over varying depth.

\section{e. Limits of the study}

Our analytical solution is limited to the mild-slope approximation and we run the numerical model with the mildslope approximation to verify the analytical model. However, the linear numerical model can be run without the mild-slope approximation, by retaining the term in $d h / d x$ in Eq. (1). We show a comparison of the phase lags and amplitude of the FLWs in those two cases (Fig. 14), and find a reasonable agreement.

In the linear numerical modeling we presented, wave groups do not break so BLWs propagate further into shallower water than they normally would. The purpose of our study was only to demonstrate the generation of FLWs as wave groups propagate over changing depth. However, the effect of shortwave breaking within a group can be taken into account to some respect since the linear model is forced directly with the wave-group amplitude and the formulations to calculate the FLW amplitude and phase [Eqs. (19)-(21)] are based on the elevation amplitude of the BLW, which depends on the wave-group amplitude [Eqs. (3) and (7)]. When validating our results with the van Noorloos (2003) laboratory experiments and output from the SWASH model, in which wave breaking occurred, we used the measured envelope of the short-wave group such that breaking is accounted for in terms of the changes to the short-wave amplitude. However, nonlinear effects, after the wave groups break, would be strong and not accounted for by the linear model. In addition, the present study only considers bichromatic unidirectional wave groups. Using the linear model, the low-frequency spectrum can be estimated by integrating the contributions of all interactions between high-frequency pairs, low frequencies and directions (Herbers et al. 1994). However, in the ocean, random waves with broad-banded spectra are typical and would need to be further investigated to fully understand all of the potential responses of the interacting short waves.

\section{Conclusions}

In this paper, we proposed an analytical solution to the linearized shallow-water equations with radiation stress forcing. This solution is verified numerically and is consistent with J03's solution. However, the approach we propose that decomposes the total long wave into forced (BLWs) and free wave components (FLWs) provides further insight into the generation mechanisms.

The continuous generation of increasingly large FLWs in response to depth gradients, as wave groups propagate toward the shore, reveals why the phase between the TLW and the wave group shifts from $180^{\circ}$ offshore and toward $90^{\circ}$ in shallower water. The combined FLW signal superimposes on the conventional (locked) BLW solution for a flat bottom (Longuet-Higgins and Stewart 1962), to form the TLW signal, usually referred to as the shoaling BLW.

The FLW component of the solution is important because, depending on phase relationships, it will either be added to or subtracted from the FLWs generated through breakpoint forcing (Symonds et al. 1982) or bound-wave release (Masselink 1995).

Acknowledgments. The authors acknowledge A. van Dongeren for providing the laboratory data (van Noorloos 2003; van Dongeren et al. 2007). This research was funded by the Bluelink Partnership: a collaboration between the Australian Department of Defence, Bureau of Meteorology and CSIRO, and by the University of Western Australia.

Data availability statement. Input SWASH files and the van Noorloos (2003) laboratory experiment measurement data are available as online supplemental material.

\section{APPENDIX A}

\section{Energy Conservation}

In this section, we verify that the model satisfies the energy balance equation. The energy balance equation can be extracted from the equations of mass and momentum conservation [Eqs. (4) and (5)].

The wave-induced potential energy and kinetic energy per unit horizontal area, integrated over the depth, are, respectively (e.g., Holthuijsen, 2007):

$$
\begin{aligned}
& E_{p}=\int_{0}^{\zeta} \rho g z d z=\frac{1}{2} \rho g \zeta^{2}, \\
& E_{k}=\int_{-h}^{0} \frac{1}{2} \rho u^{2} d z,
\end{aligned}
$$




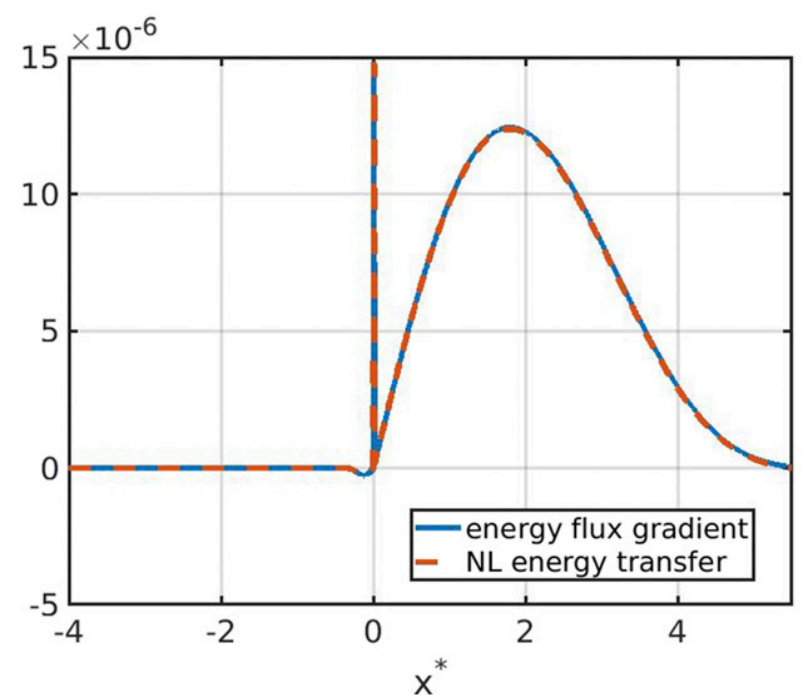

FIG. A1. Nondimensional time-averaged terms of energy balance equation vs $x^{*}$. Solid blue line is $\partial h^{*} U^{*} \zeta^{*} / \partial x^{*}$ (the energy flux gradient), dashed red line is $-U^{*} \partial S^{*} / \partial x^{*}$ (the nonlinear energy transfer term), for the step case $\left(x_{\text {step }}=0\right)$.

where $\zeta$ is the long-wave elevation and $u$ is the wave particle velocity, and $z$ is the vertical direction (positive upward). The total long-wave energy $E=E_{p}+E_{k}$. Note, the upper integration limit of the kinetic energy is 0 and not $\zeta$, because we calculate the energy to the second order, both for kinetic and potential energy (e.g., Pedlosky 2003).

Since the waves are long compared to the depth, $u$ is assumed constant over the water column, so we use the depthintegrated velocity $U$ (including Stokes drift):

$$
E_{k}=\frac{1}{2} \rho U^{2} h .
$$

To extract the energy components, we express the mass and momentum conservation equations [Eqs. (4) and (5)] in terms of potential and kinetic energy, so

$$
\begin{aligned}
\frac{\partial \zeta}{\partial t}+h \frac{\partial U}{\partial x} & =0 \\
\rho g \zeta \frac{\partial \zeta}{\partial t}+\rho g h \zeta \frac{\partial U}{\partial x} & =0 \\
\frac{1}{2} \rho g \frac{\partial \zeta^{2}}{\partial t}+\rho g h \zeta \frac{\partial U}{\partial x} & =0 \\
\frac{\partial E_{p}}{\partial t}+\rho g h \zeta \frac{\partial U}{\partial x} & =0
\end{aligned}
$$

and

$$
\begin{gathered}
\rho h \frac{\partial U}{\partial t}+\rho g h \frac{\partial \zeta}{\partial x}=-\frac{\partial S_{x x}}{\partial x}, \\
\frac{1}{2} \rho h \frac{\partial U^{2}}{\partial t}+\rho g h U \frac{\partial \zeta}{\partial x}=-U \frac{\partial S_{x x}}{\partial x}, \\
\frac{\partial E_{k}}{\partial t}+\rho g h U \frac{\partial \zeta}{\partial x}=-U \frac{\partial S_{x x}}{\partial x},
\end{gathered}
$$

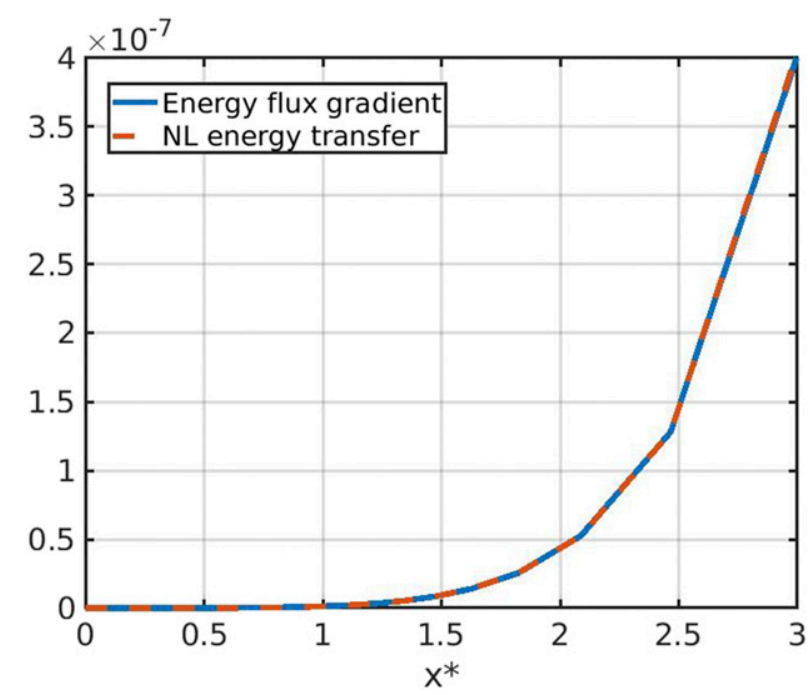

FIG. A2. Nondimensional time-averaged terms of energy balance equation vs $x^{*}$. Solid blue line is $\partial h^{*} U^{*} \zeta^{*} / \partial x^{*}$ (the energy flux gradient), dashed red line is $-U^{*} \partial S^{*} / \partial x^{*}$ (the nonlinear energy transfer term), for the slope case.

By adding the two equations, we obtain the energy balance equation for the long waves, including a source term from radiation stress:

$$
\frac{\partial E}{\partial t}+\rho g h \frac{\partial U \zeta}{\partial x}=-U \frac{\partial S_{x x}}{\partial x} .
$$

Since the long waves are sinusoidal, $\partial E / \partial t$, averaged over a wave period is zero so the time-averaged energy balance equation is

$$
\rho g h \frac{\partial U \zeta}{\partial x}=-U \frac{\partial S_{x x}}{\partial x} .
$$

The term on the left is the energy flux gradient and the term on the right represents the nonlinear (NL) energy transfers.

\section{a. Single step case}

We calculate the energy flux gradient and forcing terms in Eq. (A7) from the model, for each $x$ of the step case, to verify that the model respects the energy balance equation. The results are shown in Fig. A1.

In Fig. A1, the time-averaged energy flux gradient and nonlinear energy transfer terms of the energy balance equation [Eq. (16)] are plotted as a function of the crossshore position. Both terms are equal, showing that the forcing term (the nonlinear energy transfer term) balances the energy flux gradient.

\section{b. Slope case}

We verified in the single step case, that energy was conserved in the model. We do the same here for the slope case. We calculate the time-averaged energy flux gradient and the nonlinear energy transfer terms from the model output using Eq. (A6). 

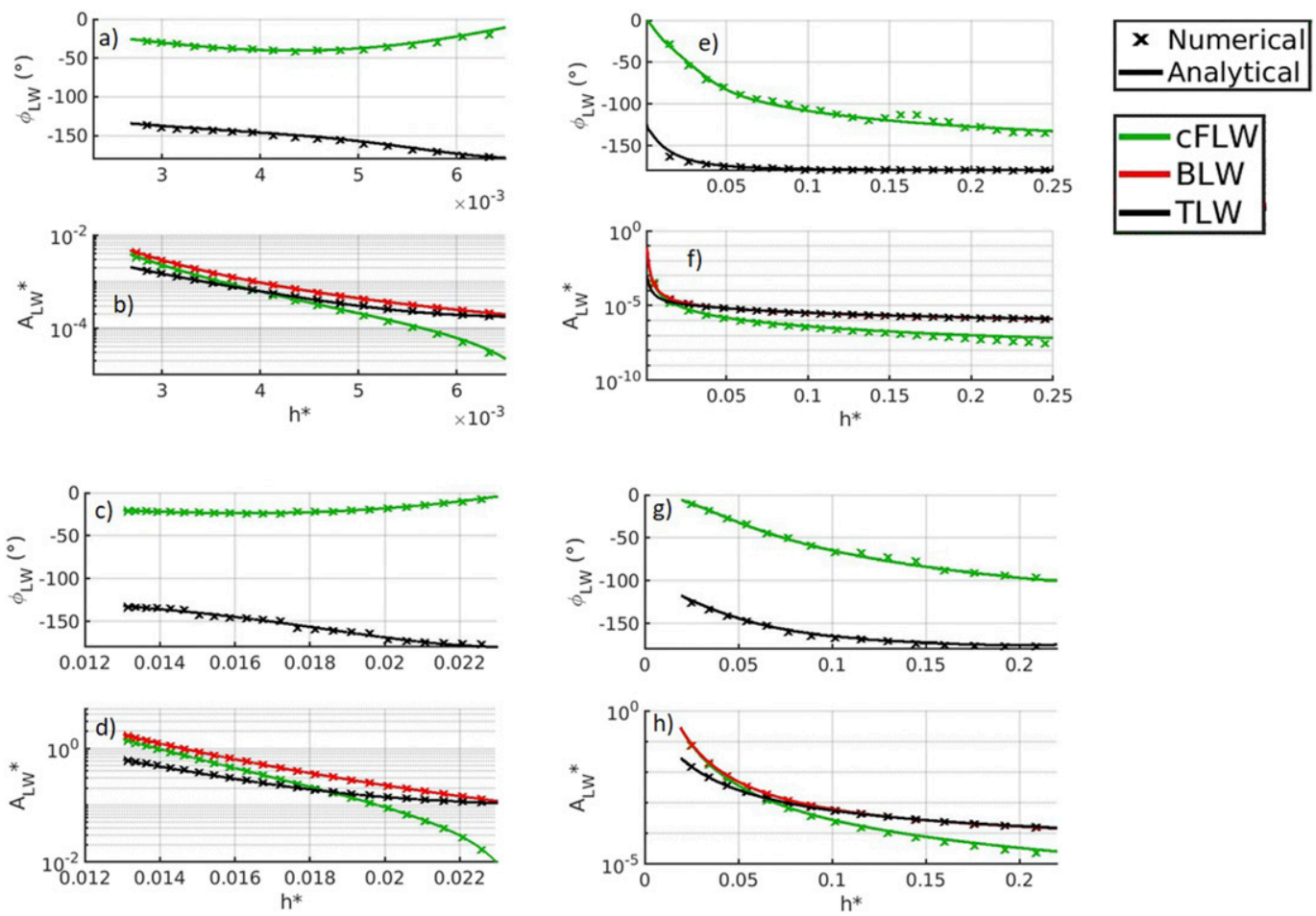

FIG. B1. (a),(c),(e),(g) Phase lags between the wave-group and the long waves and (b),(d),(f),(h) amplitude of the long wave, modeled numerically and analytically. Shown are $\beta=0.002, f=0.005 \mathrm{~Hz}$ in (a) and (b); $\beta=0.002, f=0.05 \mathrm{~Hz}$ in (c) and (d); $\beta=0.05, f=0.005 \mathrm{~Hz}$ in (e) and (f); $\beta=0.05, f=0.05 \mathrm{~Hz}$ in (g) and (h).

For the slope case, the energy flux gradient and the energy transfer terms are equal (Fig. A2), so the model correctly conserves energy. The terms are positive and increase as the depth decreases. This is similar to what Ruju et al. (2012) found using a RANS model, except here the terms keep increasing, because there is no transfer of energy back to the short waves, as short waves do not break in our model. The negative energy flux values on the seaside of the step case (Fig. A1) does not appear here, as the signal from oFLW are small compared to the iFLW. The energy flux gradient increases as depth decreases due to the energy transfer from wave groups to FLW.

\section{APPENDIX B}

\section{Numerical Model and Analytical Solutions Comparison for Different $\beta$ and Different Frequency Differences}

We run the linear model and calculate the analytical solutions for two different values of $\beta(0.002$ and 0.05$)$ and $f(0.005$ and $0.05 \mathrm{~Hz})$ in order to allow the verification of the model to cover a range of realistic cases (Fig. B1). The numerical and analytical model results agree well. We note that when the amplitude of the FLW is very small, noise can easily affect the estimation of the phase lag between the FLWs and the wave group; however, the estimation of the phase lag of the TLW is not noticeably affected. In the steep cases (Fig. B1, right column), the amplitude of the TLW increases dramatically and the phase lag of the TLW tends toward $90^{\circ}$, as depth decreases. In the mild cases (Fig. B1, left), the effect of older FLWs is visible, with the phase lag of the TLW only slowly tending toward $90^{\circ}$ as depth decreases. The values of amplitude and phase cannot be calculated close to the shoreline, as peaks of elevation cannot
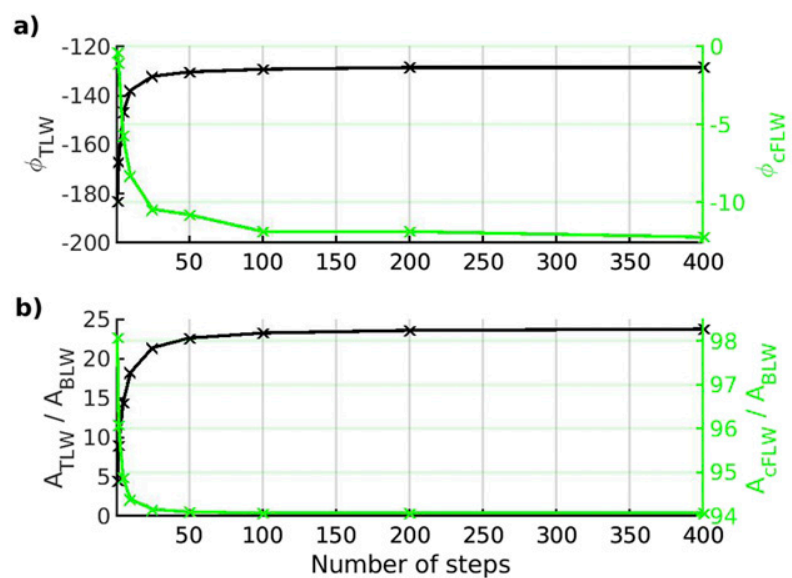

FIG. C1. (a) Phase lag between long wave and wave group and (b) amplitude of long wave relative to BLW amplitude, vs number of steps. Black: TLW, green cFLW. 

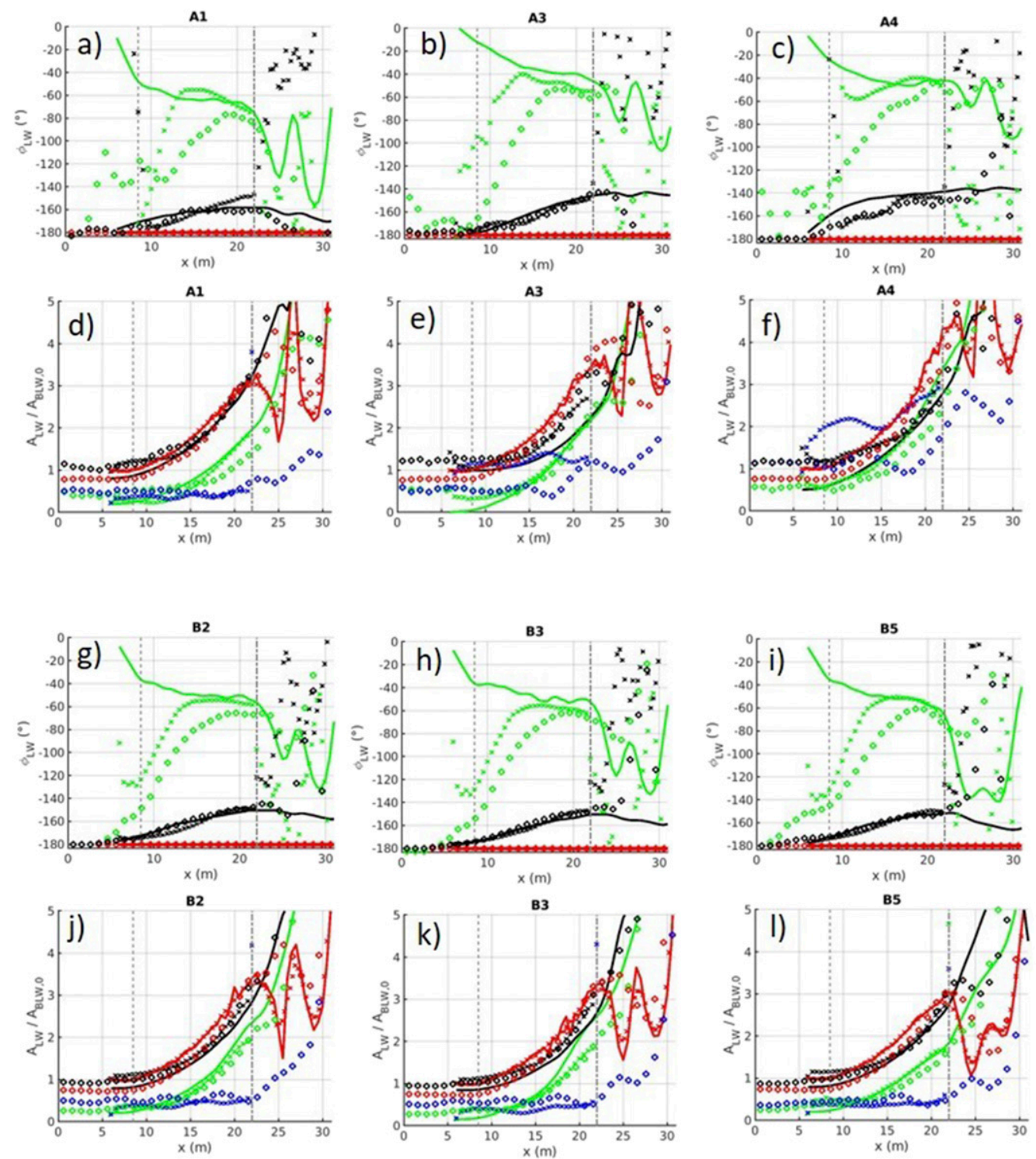

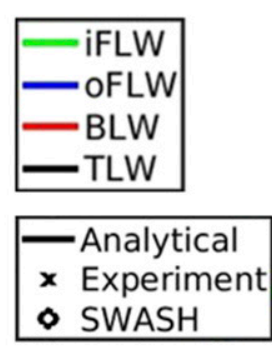

SWASH

FIG. D1. Phase lag between long wave and wave-group envelope for (a) case A2, (b) case A3, (c) case A4, (g) case B2, (h) case B3, (i) case B5, and long-wave amplitude, relative to the BLW amplitude at the start of the slope, for (d) case A1, (e) case A3, (f) case A4, (j) case B2, (k) case B3, and (1) case B5. Note that the vertical dashed gray line indicates the start of the slope, and the dash-dot gray vertical line indicates the approximate location of the short-wave breakpoint. Data are smoothed using a moving average over $3 \mathrm{~m}$.

be identified if the distance to the shoreline is less than half a wavelength.

\section{APPENDIX C}

\section{Succession of Single Steps}

We aim to test how the number of steps, over a same distance affects the amplitude of the long wave and the phase lag between the long wave and the wave group. We calculate the amplitude of a cFLW generated through a succession of single steps of equal length $\Delta x$, from 1 step to the maximum number of steps (minimum step length $\Delta x=d x$ ). The results
(Fig. C1) show that the final phase and amplitude of the long wave (on the shallowest level) vary depending on the number of steps.

If the depth variation happens in one single step, the amplitude of the (c)FLW is larger than with any other number of steps because there is only one large FLW (in antiphase with the group) instead of a superposition of smaller FLWs lagging each other. In the case of a single large step, the FLW is in antiphase with the BLW, so the amplitude of the TLW is the smallest even though the amplitude of the cFLW is the largest. However, it is important to note that, as the FLW and the BLW propagate on the flat part, the FLW propagates faster than the BLW. They behave as a bichromatic group, and when the 
TABLE D1. Amplitudes and frequencies of the short waves in the van Noorloos (2003) bichromatic wave experiments.

\begin{tabular}{lcccccc}
\hline \hline Case & $a_{1}(\mathrm{~m})$ & $a_{2}(\mathrm{~m})$ & $f_{1}(\mathrm{~Hz})$ & $f_{2}(\mathrm{~Hz})$ & $f(\mathrm{~Hz})$ & $\beta /(2 \pi f)$ \\
\hline $\mathrm{A} 1$ & 0.06 & 0.012 & 0.6714 & 0.4761 & 0.1953 & 0.0233 \\
$\mathrm{~A} 3$ & 0.06 & 0.012 & 0.6348 & 0.5127 & 0.1221 & 0.0372 \\
$\mathrm{~A} 4$ & 0.06 & 0.012 & 0.5249 & 0.0977 & 0.0977 & 0.0465 \\
$\mathrm{~B} 1$ & 0.06 & 0.012 & 0.6470 & 0.5005 & 0.1465 & 0.0310 \\
$\mathrm{~B} 2$ & 0.06 & 0.018 & 0.6470 & 0.5005 & 0.1465 & 0.0310 \\
$\mathrm{~B} 3$ & 0.06 & 0.024 & 0.6470 & 0.5005 & 0.1465 & 0.0310 \\
$\mathrm{~B} 5$ & 0.06 & 0.036 & 0.6470 & 0.5005 & 0.1465 & 0.0310 \\
\hline
\end{tabular}

crests of both long waves correspond, the total amplitude is equal to the sum of the amplitudes (of the BLW and of the FLW).

As the number of steps increases (and $d x$ decreases), the amplitude of the cFLW decreases while the amplitude of the TLW increases, and they converge toward a minimum and maximum amplitudes, respectively. In the same way, the absolute value of the phase lag of the cFLW increases and the absolute value of the phase lag of the TLW decreases, and they converge toward a maximum and minimum phase lags, respectively. The convergence shows that if $d x$ is sufficiently small (i.e., a large number of small steps are used to represent a slope), then a discrete slope can be assimilated to a continuous slope. The absolute value of the phase lag of the cFLW increases slightly, because of the presence of the smaller FLWs previously generated.

\section{APPENDIX D}

\section{Validation (Additional Cases)}

In section 6 we presented a comparison of the analytical model with numerical simulations using SWASH and laboratory experiment results, for two cases. Here (Fig. D1) we show the same results as in Fig. 11 but for the other case (Table D1).

\section{APPENDIX E}

\section{Decomposition Method}

Consider a bichromatic wave group, with an incoming wave and an outgoing wave at the frequency $f$. We assume that all wave components are progressive and of constant form. For a wave propagating in positive $x$ direction, the surface elevation $\zeta_{+}$in space $x$ and time $t$ can then be written as

$$
\frac{\zeta_{+}}{a_{+}}=\sin (k x-\omega t)
$$

with $a_{+}$the wave amplitude, $\omega$ the wave radial frequency, $k$ the wavenumber. In the same way, for a wave propagating in negative $x$ direction, the surface elevation $\zeta_{-}$can be written

$$
\frac{\zeta_{-}}{a_{-}}=\sin (k x+\omega t) \text {. }
$$

Based on this, the total wave signal can be written as

$$
\zeta=\zeta_{+}+\zeta_{-}
$$

Based on this equation we will now decompose the wave field into its two components. With two unknowns $\left[\zeta_{+}(x, t)\right.$ and $\left.\zeta_{-}(x, t)\right]$, we need one more equation to solve for the two surface elevation signals of the wave components. For all wave components, we can readily find an additional equation by taking derivatives in time or space. For example, for the incoming wave component we can find,

$$
\begin{aligned}
& \frac{\partial_{x} \zeta_{+}}{a_{+}}=-\left(k+k_{x} x\right) \cos (k x-\omega t), \\
& \frac{\partial_{x t} \zeta_{+}}{a_{+}}=-\omega\left(k+k_{x} x\right) \sin (k x-\omega t),
\end{aligned}
$$

From the above, it can be recognized that we can use the second equation to construct a system of equations to find the time signals of the two wave components.

$$
\begin{aligned}
\zeta & =\zeta_{+}+\zeta_{-} \\
\partial_{x t} \zeta & =\partial_{x t} \zeta_{+}+\partial_{x t} \zeta_{-}=-\omega\left(k+k_{x} x\right) \zeta_{+}+\omega\left(k+k_{x} x\right) \zeta_{-}
\end{aligned}
$$

Note that all surface elevation parameters are signals in the time domain. In matrix form, this system of equations reads

$$
\left[\begin{array}{cc}
1 & 1 \\
\left(k+k_{x} x\right) \omega & -\left(k+k_{x} x\right) \omega
\end{array}\right]\left[\begin{array}{c}
\zeta_{+} \\
\zeta_{-}
\end{array}\right]=\left[\begin{array}{c}
\zeta \\
\zeta_{x t}
\end{array}\right]
$$

Inverting the matrix yields the following equations for the two wave components,

$$
\begin{aligned}
& \zeta_{+}=\frac{1}{2}\left[\zeta+\frac{1}{\left(k+k_{x} x\right) \omega} \partial_{x t} \zeta\right] \\
& \zeta_{-}=\frac{1}{2}\left[\zeta-\frac{1}{\left(k+k_{x} x\right) \omega} \partial_{x t} \zeta\right]
\end{aligned}
$$

This method is similar to the method proposed by Guza et al. (1985), but it has the advantage of being valid on a slope because it accounts for a wavenumber varying with $x$ and it can be used when current velocity measurements are not available.

\section{REFERENCES}

Battjes, J. A., H. J. Bakkenes, T. T. Janssen, and A. R. van Dongeren, 2004: Shoaling of subharmonic gravity waves. J. Geophys. Res., 109, C02009, https://doi.org/10.1029/2003JC001863.

Beetham, E. P., and P. S. Kench, 2011: Field observations of infragravity waves and their behaviour on rock shore platforms. Earth Surf. Processes Landforms, 36, 1872-1888, https:// doi.org/10.1002/esp.2208.

Bertin, X., and Coauthors, 2018: Infragravity waves: From driving mechanisms to impacts. Earth-Sci. Rev., 117, 774-779, https:// doi.org/10.1016/j.earscirev.2018.01.002.

_ , K. Martins, A. de Bakker, T. Chataigner, T. Guérin, T. Coulombier, and O. de Viron, 2020: Energy transfers and reflection of infragravity waves at a dissipative beach under storm waves. J. Geophys. Res. Oceans, 125, e2019JC015714, https://doi.org/10.1029/2019JC015714.

Buckley, M. L., R. J. Lowe, J. E. Hansen, and A. R. van Dongeren, 2015: Dynamics of wave setup over a steeply-sloping fringing 
reef. J. Phys. Oceanogr., 45, 3005-3023, https://doi.org/10.1175/ JPO-D-15-0067.1.

Contardo, S., and G. Symonds, 2013: Infragravity response to variable wave forcing in the nearshore. J. Geophys. Res. Oceans, 118, 7095-7106, https://doi.org/10.1002/2013JC009430.

— time-varying breakpoint with real wave conditions. J. Coast. Res., 75, 836-840, https://doi.org/10.2112/SI75-168.1.

$\longrightarrow,-$, and F. Dufois, 2018: Breakpoint forcing revisited: Phase between forcing and response. J. Geophys. Res. Oceans, 123, 1354-1363, https://doi.org/10.1002/2017JC013138.

de Bakker, A. T. M., J. A. Brinkkemper, F. van der Steen, M. F. S. Tissier, and B. G. Ruessink, 2016: Cross-shore sand transport by infragravity waves as a function of beach steepness. J. Geophys. Res. Earth Surf., 121, 1786-1799, https://doi.org/10.1002/2016JF003878.

Elgar, S., T. H. C. Herbers, M. Okihiro, J. Oltman-Shay, and R. T. Guza, 1992: Observations of infragravity waves. J. Geophys. Res., 97, 15 573-15 577, https://doi.org/10.1029/92JC01316.

García-Medina, G., H. T. Özkan-Haller, R. A. Holman, and P. Ruggiero, 2017: Large runup controls on a gently sloping dissipative beach. J. Geophys. Res. Oceans, 122, 5998-6010, https://doi.org/10.1002/2017JC012862.

Gomes, E. R., R. P. Mulligan, K. L. Brodie, and J. E. McNinch, 2016: Bathymetric control on the spatial distribution of wave breaking in the surf zone of a natural beach. Coast. Eng., 116, 180-194, https://doi.org/10.1016/j.coastaleng.2016.06.012.

Guérin, T., A. de Bakker, and X. Bertin, 2019: On the bound wave phase lag. Fluids, 4, 152, https://doi.org/10.3390/fluids4030152.

Guza, R. T., E. B. Thornton, and R. A. Holman, 1985: Swash on steep and shallow beaches. 19th Int. Conf. on Coastal Engineering, Houston, TX, ASCE, 708-723, https://doi.org/ 10.9753/icce.v19.48.

Herbers, T. H. C., S. Elgar, R. T. Guza, T. H. C. Herbers, S. Elgar, and R. T. Guza, 1994: Infragravity-frequency $(0.005-0.05 \mathrm{~Hz})$ motions on the shelf. Part I: Forced waves. J. Phys. Oceanogr., 24, 917-927, https://doi.org/10.1175/1520-0485(1994)024<0917: IFHMOT $>2.0 . \mathrm{CO} ; 2$.

Holthuijsen, L. H., 2007: Waves in Oceanic and Coastal Waters. Cambridge University Press, 404 pp.

Inch, K., M. Davidson, G. Masselink, and P. Russell, 2017: Observations of nearshore infragravity wave dynamics under high energy swell and wind-wave conditions. Cont. Shelf Res., 138, 19-31, https://doi.org/10.1016/j.csr.2017.02.010.

Janssen, T. T., J. A. Battjes, and A. R. van Dongeren, 2003: Long waves induced by short-wave groups over a sloping bottom. J. Geophys. Res., 108, 3252, https://doi.org/10.1029/2002JC001515.

Lara, J. L., A. Ruju, and I. J. Losada, 2010: Reynolds averaged Navier-Stokes modelling of long waves induced by a transient wave group on a beach. Proc. Roy. Soc., 467A, 1215-1242, https://doi.org/10.1098/RSPA.2010.0331.

Le Mehauté, B., 1976: An Introduction to Hydrodynamics and Water Waves. Springer, $323 \mathrm{pp}$.

Li, S., Z. Liao, Y. Liu, and Q. Zou, 2020: Evolution of infragravity waves over a shoal under non-breaking conditions. J. Geophys. Res. Oceans, 125, e2019JC015864, https:// doi.org/10.1029/2019JC015864.

List, J. H., 1992: A model for the generation of two-dimensional surf beat. J. Geophys. Res., 97, 5623, https://doi.org/10.1029/ 91JC03147.

Longuet-Higgins, M. S., and R. W. Stewart, 1962: Radiation stress and mass transport in gravity waves, with application to 'surf beats.' J. Fluid Mech., 13, 481-504, https://doi.org/10.1017/ S0022112062000877.
Masselink, G., 1995: Group bound long waves as a source of infragravity energy in the surf zone. Cont. Shelf Res., 15, 15251547, https://doi.org/10.1016/0278-4343(95)00037-2.

—, M. Tuck, R. McCall, A. van Dongeren, M. Ford, and P. Kench, 2019: Physical and numerical modeling of infragravity wave generation and transformation on coral reef platforms. J. Geophys. Res. Oceans, 124, 1410-1433, https:// doi.org/10.1029/2018JC014411.

Mei, C. C., and C. Benmoussa, 1984: Long waves induced by shortwave groups over an uneven bottom. J. Fluid Mech., 139, 219235, https://doi.org/10.1017/S0022112084000331.

Mendes, D., J. P. Pinto, A. A. Pires-Silva, and A. B. Fortunato, 2018: Infragravity wave energy changes on a dissipative barred beach: A numerical study. Coast. Eng., 140, 136-146, https:// doi.org/10.1016/j.coastaleng.2018.07.005.

Molin, B., 1982: On the generation of long period second order free waves due to changes in the bottom profile. Ship Hydromechanics and Structures Rep. 68, 28 pp., http://resolver.tudelft.nl/ uuid:50c0c204-45fd-41f8-b22c-778508726d33.

Moura, T., and T. E. Baldock, 2017: Remote sensing of the correlation between breakpoint oscillations and infragravity waves in the surf and swash zone. J. Geophys. Res. Oceans, 122, 31063122, https://doi.org/10.1002/2016JC012233.

— , and - 2019: The influence of free long wave generation on the shoaling of forced infragravity waves. J. Mar. Sci. Eng., 7, 305, https://doi.org/10.3390/jmse7090305.

Nagase, S., and M. Mizuguchi, 1997: Laboratory experiment on long wave generation by time-varying breakpoint. 25th Proc. Coastal Engineering Conf., Orlando, FL, ASCE, 1307-1320, https://doi.org/10.1061/9780784402429.102.

Nielsen, P., 2017: Surf beat "shoaling." Coastal Dynamics, Helsing $\varnothing \mathrm{r}, 443-450$.

— terms of transient forced long waves. Coast. Eng., 57, 71-73, https://doi.org/10.1016/j.coastaleng.2009.09.003.

Okihiro, M., R. T. Guza, and R. J. Seymour, 1992: Bound infragravity waves. J. Geophys. Res., 57, 11 453-11469, https:// doi.org/10.1029/92JC00270.

Pedlosky, J., 2003: Waves in the Ocean and Atmosphere: Introduction to Wave Dynamics. Springer, $259 \mathrm{pp}$.

Péquignet, A. C. N., J. M. Becker, and M. A. Merrifield, 2014: Energy transfer between wind waves and low-frequency oscillations on a fringing reef, Ipan, Guam. J. Geophys. Res. Oceans, 119, 67096724, https://doi.org/10.1002/2014JC010179.

Pomeroy, A., R. Lowe, G. Symonds, A. van Dongeren, and C. Moore, 2012: The dynamics of infragravity wave transformation over a fringing reef. J. Geophys. Res., 117, C11022, https://doi.org/10.1029/2012JC008310.

Reniers, A. J. H. M., A. R. van Dongeren, J. A. Battjes, and E. B. Thornton, 2002: Linear modeling of infragravity waves during Delilah. J. Geophys. Res., 107, 3137, https://doi.org/10.1029/ 2001JC001083.

Rijnsdorp, D. P., P. B. Smit, and M. Zijlema, 2014: Non-hydrostatic modelling of infragravity waves under laboratory conditions. Coast. Eng., 85, 30-42, https://doi.org/10.1016/j.coastaleng.2013.11.011.

——, G. Ruessink, and M. Zijlema, 2015: Infragravity-wave dynamics in a barred coastal region, a numerical study. J. Geophys. Res. Oceans, 120, 4068-4089, https://doi.org/ 10.1002/2014JC010450.

Roelvink, J. A., H. A. A. Petit, and J. K. Kostense, 1993: Verification of a one-dimensional surfbeat model against laboratory data. Coastal Engineering Proc., 1, (23), 960-973, https://doi.org/10.9753/icce.v23.\%25p. 
Ruessink, B. G., 1998: Bound and free infragravity waves in the nearshore zone under breaking and nonbreaking conditions. J. Geophys. Res., 103, 12 795-12 805, https://doi.org/10.1029/ 98JC00893.

Ruju, A., J. L. Lara, and I. J. Losada, 2012: Radiation stress and low-frequency energy balance within the surf zone: A numerical approach. Coast. Eng., 68, 44-55, https://doi.org/ 10.1016/j.coastaleng.2012.05.003.

,-- , and $—, 2019$ : Numerical assessment of infragravity swash response to offshore wave frequency spread variability. J. Geophys. Res. Oceans, 124, 6643-6657, https://doi.org/ 10.1029/2019JC015063.

Schäffer, H. A., 1993: Infragravity waves induced by short-wave groups. J. Fluid Mech., 247, 551-588, https://doi.org/10.1017/ S0022112093000564.

Smit, P., M. Zijlema, and G. Stelling, 2013: Depth-induced wave breaking in a non-hydrostatic, near-shore wave model. Coast. Eng., 76, 1-16, https://doi.org/10.1016/ j.coastaleng.2013.01.008.

Svendsen, I. A., and J. B. Hansen, 1977: The wave height variation for regular waves in shoaling water. Coast. Eng., 1, 261-284, https://doi.org/10.1016/0378-3839(77)90018-7.

Symonds, G., D. A. Huntley, and A. J. Bowen, 1982: Two-dimensional surf beat: Long wave generation by a time-varying breakpoint. J. Geophys. Res., 87, 492, https://doi.org/10.1029/ JC087iC01p00492.

Torres-Freyermuth, A., J. L. Lara, and I. J. Losada, 2010: Numerical modelling of short- and long-wave transformation on a barred beach. Coast. Eng., 57, 317-330, https://doi.org/ 10.1016/j.coastaleng.2009.10.013.

van Dongeren, A., A. Reniers, J. Battjes, and I. Svendsen, 2003: Numerical modeling of infragravity wave response during DELILAH. J. Geophys. Res., 108, 3288, https://doi.org/ 10.1029/2002JC001332.

—, J. Battjes, T. Janssen, J. van Noorloos, K. Steenhauer, G. Steenbergen, and A. Reniers, 2007: Shoaling and shoreline dissipation of low-frequency waves. J. Geophys. Res., 112, C02011, https://doi.org/10.1029/2006JC003701.

van Leeuwen, P. J., 1992: Low frequency wave generation due to breaking wind waves. Natural Physical Sources of Underwater Sound, Springer, 277-304.

van Noorloos, J. C., 2003: Energy transfer between short wave groups and bound long waves on a plane slope. M.S. thesis, Dept. of Civil Engineering and Geoscience, Delft University of Technology, $84 \mathrm{pp}$.

Zhang, Q., E. A. Toorman, and J. Monbaliu, 2020: Shoaling of bound infragravity waves on plane slopes for bichromatic wave conditions. Coast. Eng., 158, 103684, https://doi.org/ 10.1016/j.coastaleng.2020.103684.

Zijlema, M., G. Stelling, and P. Smit, 2011: SWASH: An operational public domain code for simulating wave fields and rapidly varied flows in coastal waters. Coast. Eng., 58, 9921012, https://doi.org/10.1016/j.coastaleng.2011.05.015.

Zou, Q., 2004: A simple model for random wave bottom friction and dissipation. J. Phys. Oceanogr., 34, 1459-1467, https://doi.org/ 10.1175/1520-0485(2004)034<1459:ASMFRW>2.0.CO;2. 